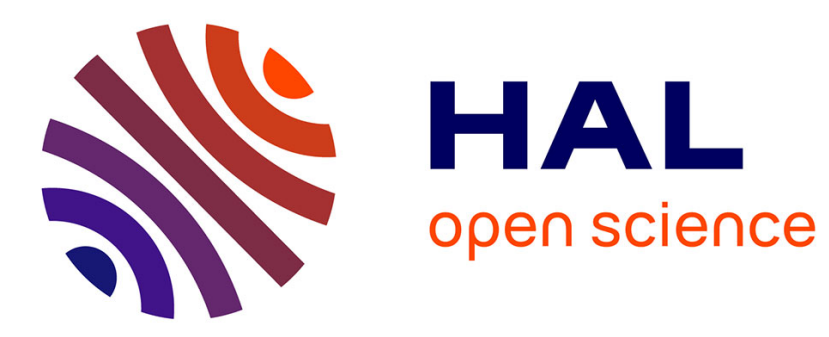

\title{
SR3: secure resilient reputation-based routing
}

Karine Altisen, Raphaël Jamet, Stéphane Devismes, Pascal Lafourcade

\section{To cite this version:}

Karine Altisen, Raphaël Jamet, Stéphane Devismes, Pascal Lafourcade. SR3: secure resilient reputation-based routing. Wireless Networks, 2017, 23 (7), pp.2111 - 2133. 10.1007/s11276-0161273-4 . hal-01707698

\section{HAL Id: hal-01707698 \\ https://hal.science/hal-01707698}

Submitted on 13 Feb 2018

HAL is a multi-disciplinary open access archive for the deposit and dissemination of scientific research documents, whether they are published or not. The documents may come from teaching and research institutions in France or abroad, or from public or private research centers.
L'archive ouverte pluridisciplinaire HAL, est destinée au dépôt et à la diffusion de documents scientifiques de niveau recherche, publiés ou non, émanant des établissements d'enseignement et de recherche français ou étrangers, des laboratoires publics ou privés. 


\title{
SR3: Secure Resilient Reputation-based Routing
}

Karine Altisen - Stéphane Devismes •

Raphaël Jamet · Pascal Lafourcade

\begin{abstract}
In this paper, we propose SR3 (which means Secure Resilient Reputationbased Routing), a secure and resilient algorithm for convergecast routing in WSNs (Wireless Sensor Networks). SR3 uses lightweight cryptographic primitives to achieve data confidentiality and unforgeability. Security of SR3 has been proven formally using two verification tools: CryptoVerif and Scyther. We made simulations to show the resiliency of SR3 against various scenarios, where we mixed selective forwarding, blackhole, wormhole, and Sybil attacks. We compared our solution to several routing algorithms of the literature. Our results show that the resiliency accomplished by SR3 is drastically better than the one achieved by those protocols, especially when the network is sparse. Moreover, unlike previous solutions, SR3 self-adapts after compromised nodes suddenly change their behavior.
\end{abstract}

Keywords Wireless sensor networks · Routing · Security · Resiliency

\section{Introduction}

Nowadays, there is a growing interest in WSNs. WSNs are multi-hop mesh networks made of numerous small battery-powered sensors that generate data about the environment (e.g., temperature) and use them for specific services (e.g., emit an alarm when the surrounding temperature is too high). Moreover, they embed wireless communication capabilities allowing them to exchange data. The low capabilities of the sensors, their wireless communications, and the fact that they are deployed in open areas make them prone to attacks.

Routing is a crucial issue in WSNs. Here, we consider a routing scheme called convergecast routing. In this problem, a node is distinguished as the sink and all

A preliminary version of this paper appeared in [3].

K. Altisen, S. Devismes and R. Jamet

VERIMAG, Université de Grenoble

E-mail: Firstname.Lastname@imag.fr

P. Lafourcade

LIMOS, Clermont Université

E-mail: Firstname.Lastname@imag.fr 
non-sink nodes, called here source nodes, must be able to transmit data to the sink on request or according to an a priori unknown schedule. The sink can be arbitrarily far (in terms of hops) from other nodes. Typically, in WSNs, source nodes are sensors and the sink is a base station that is linked to another network, like a gateway.

A routing protocol in a WSN may have to face many kinds of attacks. Here, we consider the critical scenario, where some sensors are compromised and controlled by an attacker. In particular, such an internal attacker has access to all secret and received information of the compromised nodes.

The attacker can impact the routing protocol at two main levels:

Message Level: First, he can attack the data message to learn secret information, i.e., violate the data confidentiality, as this property consists in guaranteeing that data remain secret between the source and destination.

He can also make the sink deliver incorrect information, i.e., violate the integrity of data messages. Integrity guarantees that the destination is able to detect whether the data inside a message have been modified.

Moreover, the attacker can act against the authentication of the nodes. Authenticity guarantees that the destination is able to detect whether the alleged source in a message is the true one.

Routing Level: Secondly, the attacker can affect the routing scheme itself, e.g., he may prevent data from being delivered by the sink (leading to degrade the quality of service, essentially the delivery rate), or create congestion by increasing the load in all or part of the network (leading to reduce the lifetime of the network). Such an attack can also evolve over time, e.g., the attacker can attract traffic to a given intruder node using various means. Thanks to that, the intruder node (called a sinkhole) will have more impact for further malicious actions.

Related Work. Numerous solutions have been introduced to cope with attacks on data. The confidentiality, authenticity, and integrity properties are mainly guaranteed using cryptographic mechanisms. However, the choice of the cryptographic primitives should be led by the inherent constraint of WSNs. WSNs being limited in terms of resource and power, lightweight cryptographic mechanisms [18] are mandatory. An example of such a mechanism is elliptic curve cryptography $[26,29]$. In contrast, classical asymmetric cryptography, e.g., $R S A$, should be excluded due to its computational cost.

Using such lightweight cryptographic primitives, routing protocols implementing some security properties have been proposed, e.g., $\mu$-Tesla $[31,27]$ is a broadcast authentication protocol that enables receivers of the broadcast data to verify that these data really originate from the alleged sources. $\mu$-Tesla has a low communication and computation overhead, scales to a large number of receivers, and tolerates message loss.

Although it is not strictly a routing protocol, SPINS [31] is a set of tools for routing, which provides security guarantees without using any costly operations: $\mu$-Tesla is one part of SPINS; the other part is SNEP, a message format that guarantees various security properties, like authenticity and confidentiality, using few additional bits per message.

Some other protocols, called secure route discovery protocols, have been introduced $[30,24]$ to help securing routing. Actually, they compute a valid route (i.e., 
the computed path exists in the network) between the source and destination, and for some of them (e.g., [30]), they guarantee that nodes in the chosen route achieved a certain security level, e.g., the integrity of the discovered route, which means that the computed route has been effectively traversed during the discovery process.

However, all aforementioned solutions do not use specific strategies to combat attacks at the routing level, e.g., selected forwarding, blackhole, etc. Specific approaches have been proposed to maintain a good quality of service in presence of insiders, that drop all or part of messages. For example, the notion of resiliency has been introduced in $[21,22]$ as the ability of a network to "continue operating" in presence of compromised nodes, i.e., the capacity of a network to endure and overcome internal attacks. For example, a resilient routing protocol should achieve a "graceful degradation" in the delivery rate with increasing the number of compromised nodes. In $[19,22]$, authors experimentally analyze the resiliency of several classical routing techniques, e.g., random walk [2], gradient-based routing [33], geographic routing [14]. The experimental results show that these solutions are weak in terms of resiliency. Then, they propose several resilient variants of the gradientbased routing; this latter routes messages following a Destination Oriented Directed Acyclic Graph (DODAG). Mainly, they introduce randomization and duplication in that protocol. As a result, the proposed patches drastically increase the delivery rate when the network is subject to selective forwarding or blackhole attacks. However, in their simulations, they always assume that the DODAG is available and not attacked by insiders. Moreover, they mainly consider dense networks in their simulations, e.g., networks with average degree around 30 .

Contribution. This paper deals with convergecast routing in WSNs, where all source nodes have several messages to route. We propose a Secure, Resilient, and Reputation-based Routing algorithm, called SR3. This protocol is a reinforced random walk that is partially determinized using a reputation mechanism.

SR3 uses lightweight cryptographic primitives: symmetric cryptography (precisely, authenticated encryption [9]), nonces, and hash functions. Thanks to these primitives, it achieves interesting security properties, including data confidentiality and unforgeability, this latter property implies integrity and authenticity of the data. We prove the desirable security properties achieved by SR3 in the computational model using the formal tool CryptoVerif [12]. We also prove in the symbolic model the secrecy of data and authentication of nodes using the tool Scyther [15].

Then, we show the resiliency of SR3 against various scenarios, where we mixed selective forwarding, blackhole, wormhole, and Sybil attacks. The resiliency of our algorithm is mainly captured using the delivery rate and some fairness measure. Our simulation results show in particular that unlike previous solutions, SR3 selfadapts when compromised nodes change their behavior (e.g, an interesting case is when a compromised node behaves well to attract the traffic and then suddenly decide to drop all received messages). We compare our solution to several routing algorithms of the literature, including resilient ones. Our simulations show that the resiliency accomplished by SR3 is drastically better than the one achieved by those protocols, especially when the network is sparse.

A shortcoming of our solution is the number of hops to reach the destination, as it is usually greater than other solutions of the literature. However, in our 
experiments, we observed that this complexity remains sublinear in the number of nodes.

Note also that our solution is reactive (i.e., in absence of data to route the protocol eventually stops.), has a low overhead in terms of communications, and does not use any underlying infrastructure, such as spanning tree or DODAG. Hence, SR3 is well-suited for WSNs.

Roadmap. The remainder of the paper is organized as follows. In the next section, we present our routing algorithm, SR3. Section 3 deals with the automatic proof of the security properties of SR3 using CryptoVerif [12] and Scyther [15]. In Section 4, we present experimental results that show the resiliency of SR3. Section 5 is dedicated to concluding remarks.

\section{SR3}

The formal code of our routing protocol, SR3, is given in Algorithms 1 and 2. Below, we identify the assumptions we made about networks. Then, we informally explain the behavior of SR3.

\subsection{Assumptions}

We consider arbitrary connected networks with bidirectional links, although we will focus on Unit Disk Graphs (UDG) in simulations. Each node $p$ has a unique ID (to simplify, we shall identify any node with its identifier, whenever convenient) and knows the set of its neighbors, $\mathcal{N} e i g_{p}$ - this latter assumption will be relaxed, when considering Sybil attacks.

Networks are made of one sink, which is the data collector, and numerous source nodes. The source nodes are sensors, and consequently are limited in terms of memory, computational power, and battery. Sensors are non-trustworthy since they are vulnerable to physical attacks and an adversary can compromise them. In contrast, the sink is assumed to be robust and powerful in terms of memory, computation, and energy. So, we assume that it cannot be compromised.

All nodes have access to a lightweight cryptography library (hash function, symmetric encryption, and random number generation). Each source node shares a symmetric key with the sink. Moreover, we assume that all source nodes have several data to route; however, the scheduling of the data generation is a priori unknown. Finally, there is no time synchronization between nodes.

\subsection{Overview}

Randomization is interesting to obtain resilient solutions because it generates behaviors unpredictable by an attacker. However, note that the "classical" uniform random walk, where a node chooses the next hop uniformly at random among its neighbors, is known to be inefficient even against a small number of compromised nodes [21]. So, we designed SR3 rather as a reinforced random walk, based on a reputation mechanism. The idea is to locally increase the probability of a neighbor 
to be chosen at the next hop, if it behaves well. Such a reputation mechanism is based on acknowledgments. We propose a scheme in which if a process receives a valid acknowledgment, it has the guarantee that the sink actually delivered the corresponding data message. Hence, upon receiving such an acknowledgment, a process can legitimately increase its confidence on the neighbor to which it previously sent the corresponding data message. Therefore, eventually all honest nodes preferably choose their highly-reputed neighbors, and so the data messages tend to follow paths that successfully route data to the sink.

\subsection{Reputation Mechanism}

To implement our reputation mechanism, we identify each data message (tagged MSG in the algorithm) with a nonce, i.e., an unpredictable random number that should remain secret between the source and sink until the delivery of the data message.

Assume that node $v$ initiates the routing of some value Data. It first generates a nonce $N_{v}$ (New_Nonce(), Line 1). Then, it encrypts in a ciphertext $C$ the concatenation of Data and $N_{v}$ using the key $k_{v s}$ it shares with the sink only $\left(\mathrm{E}_{k_{v s}}\left(\left\langle\right.\right.\right.$ Data, $\left.\left.N_{v}\right\rangle\right)$, Line 3). Then, both $C$ and the identifier of $v$ (in plaintext) are routed to the sink, and only the sink is able to decrypt $C$. So, upon receiving the data message, the sink decrypts $C$ using $k_{v s}$, delivers Data, and sends back to $v$ an acknowledgment ACK containing $N_{v}$ (Lines 36-39). Finally, if $v$ receives this acknowledgment, it has the guarantee that Data has been delivered, thanks to $N_{v}$.

Now, during the routing, a compromised relay node can blindly modify the encrypted part of the message. To prevent the sink from delivering erroneous data, we assume a (symmetric) authenticated encryption scheme [9], which guarantees confidentiality, authenticity, and integrity of encrypted information. This way, when receiving a message, the sink checks the validity of the message by decrypting the ciphertext using the key keys[o] of the alleged source o of the message $\left(\mathrm{E}_{k e y s[o]}^{-1}(C)\right.$, Line 36). If the ciphertext has been modified, then $\mathrm{E}_{\text {keys }[o]}^{-1}(C)=\perp$ (with overwhelming probability) and the message is simply discarded. Similarly, if a compromised node has modified the plaintext identifier in the message, then either keys $[o]$ is undefined, or the sink decrypts the ciphertext with a wrong key (in this latter case again, $\mathrm{E}_{\text {keys }[o]}^{-1}(C)=\perp$ ). So, in both cases the message is also discarded.

Upon receiving an acknowledgment, if the receiving node $v$ is the initiator of the corresponding data message $m, v$ can conclude that $m$ has been delivered. In that case, $v$ should reinforce the probability associated to the neighbor to which it previously sent $m$. To achieve that, we proceed as follows: when $v$ initiates the routing of $m, v$ saves in the list $L_{S e n t}$ the nonce stored in $m$, together with the identifier of the neighbor to which $v$ sends $m$ ( $L_{\text {Sent }}$ is appended in Line 5 using $\odot$, this latter operator is defined below). Hence, on reception of an acknowledgment, $v$ checks (in Line 20) if it is the destination of the acknowledgment and if the nonce

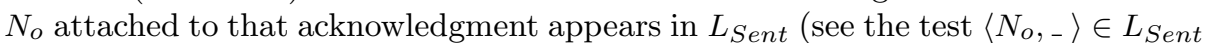
in Line 20). ${ }^{1}$ In that case, $v$ gets back the corresponding neighbor from the list $\left(\operatorname{GET}\left(L_{\text {Sent }}, N_{o}\right)\right.$, Line 21$)$, increases its confidence on that neighbor (Line 22,

\footnotetext{
1 "_" means "any value". So, $\left\langle N_{O},_{-}\right\rangle$is any record whose left value is $N_{o}$.
} 


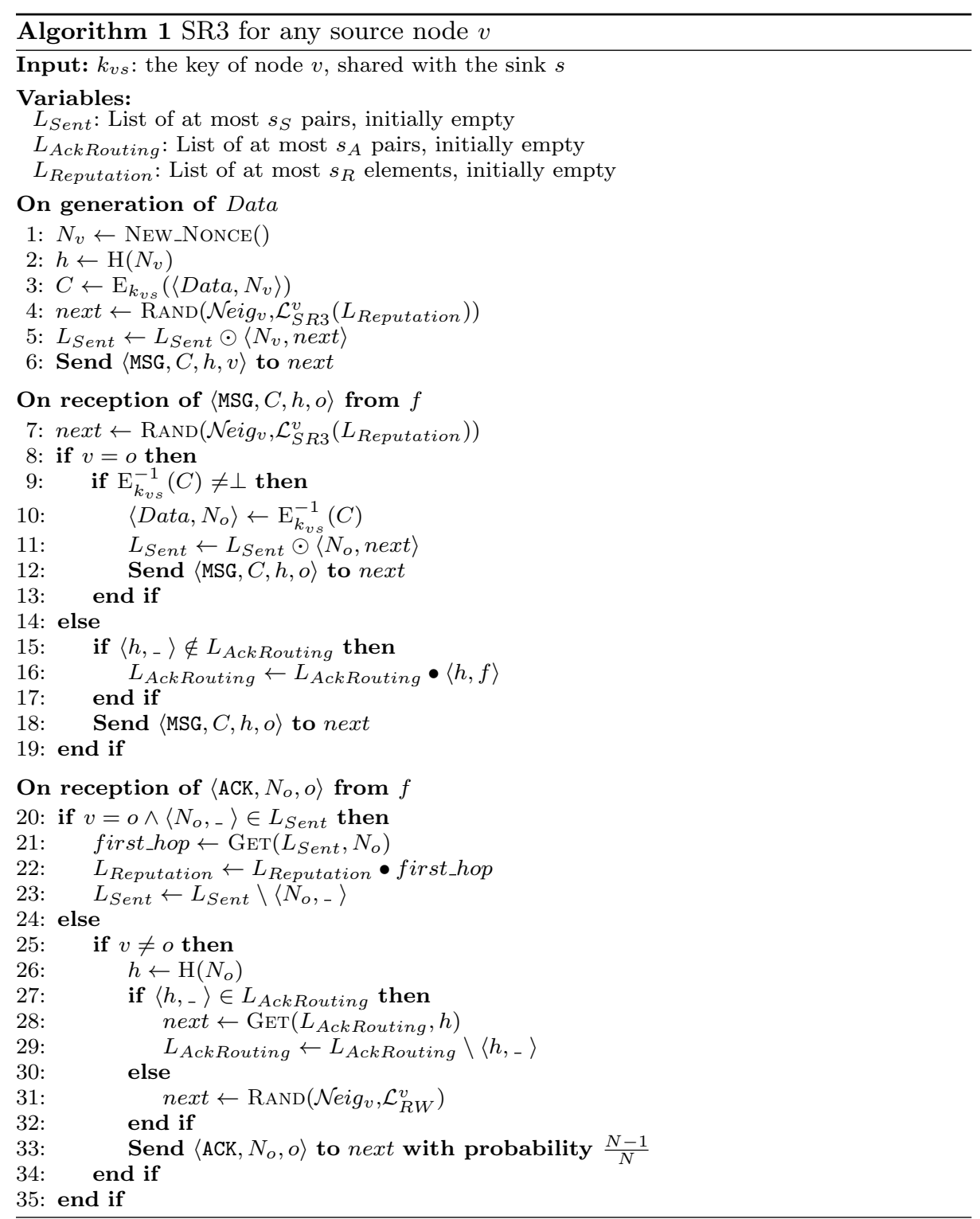

further details about increasing the confidence are given in Subsection 2.4), and removes the record from $L_{\text {Sent }}\left(L_{\text {Sent }} \backslash\left\langle N_{o},_{-}\right\rangle\right.$, Line 23). (If $v$ is the destination of the acknowledgment, but $N_{o}$ does not appear in $L_{S e n t}$, the acknowledgment is simply discarded.)

Due to the memory limitations, $L_{S e n t}$ must have a maximum size, ${ }^{2} s_{Q}$. If a node $v$ has some new data to route and $L_{S e n t}$ is full (that is, it contains $s_{Q}$ elements), then the oldest element is removed from the list to make room for the

\footnotetext{
2 All lists used in SR3 are of bounded size. We made several experiments to choose the appropriate bounds, see Subsection 4.2.
} 


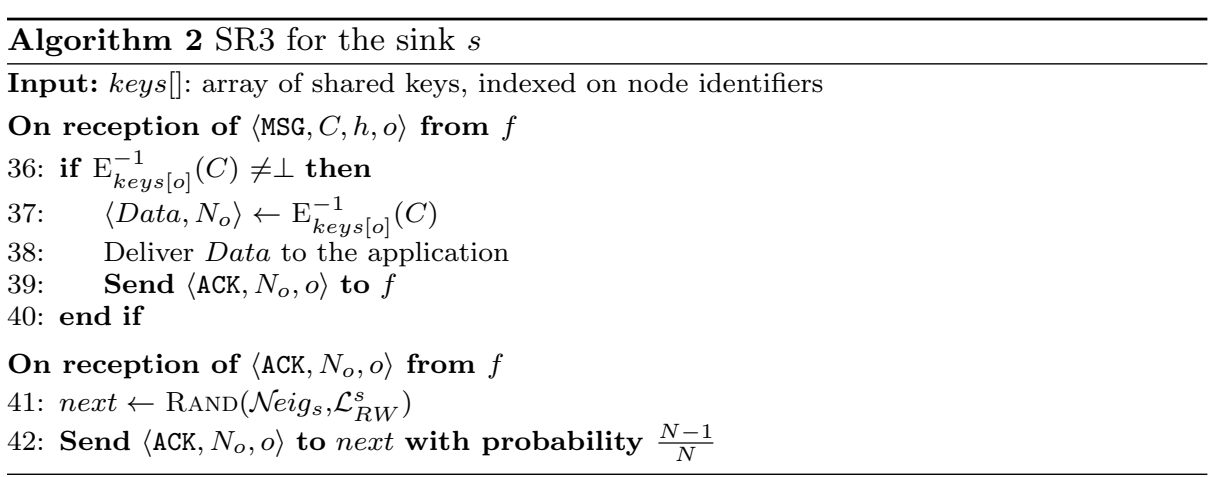

new one. A side effect is that records about lost messages or of messages whose acknowledgment has been lost are eventually removed from $L_{\text {Sent }}$.

Note that it may happen that some data message $m$ comes back to the node $v$ from which it originates because $m$ followed a cycle in the network. In this case (Lines 8-13), the validity of $m$ is checked, and if so, the routing process of $m$ is restarted. Since the old entry in $L_{\text {Sent }}$ is not relevant anymore, it is simply replaced by the new one.

Consequently, the concatenation of $\langle x, y\rangle$ to the list $L$ using $\odot$ works as follows: first, if $L$ contains any pair with a left member equal to $x$, that pair is removed from $L$; then, if $L$ is (still) full, the rightmost pair is removed; finally, $\langle x, y\rangle$ is inserted on the left side of the list. Note that, using $\odot$, any left member of a pair in the list is unique.

\subsection{Compute the Reputation}

To choose the next hop of some data message, a node performs a random choice among its neighbors, weighted according to their reputation (see Lines 4 and 7).

The reputation of a neighbor actually corresponds to the number of occurrences of its identifier in the list $L_{\text {Reputation }}$ : each time a node $v$ wants to reinforce the reputation of some neighbor $u$, it simply adds an occurrence of $u$ into its list (Line 22).

Our reputation mechanism is implemented using the probability law denoted by $\mathcal{L}_{S R 3}^{v}\left(L_{\text {Reputation }}\right)$ : Let $X$ be a random variable taking value in $\mathcal{N}$ eigv ; the law $\mathcal{L}_{S R 3}^{v}\left(L_{\text {Reputation }}\right)$ is defined, $\forall x \in \mathcal{N}$ eig, , by:

$$
\operatorname{Pr}(X=x)=\frac{\left|L_{\text {Reputation }}\right| x+\delta_{v}^{-1}}{\left|L_{\text {Reputation }}\right|+1}
$$

Where $\delta_{v}$ is the degree of $v,\left|L_{\text {Reputation }}\right|$ is the number of elements in $L_{\text {Reputation }}$, and $\left|L_{\text {Reputation }}\right|_{x}$ is the number of occurrences of $x$ in $L_{\text {Reputation. }}$. Hence, when $v$ wants to route a data message, it chooses its next destination according to $\mathcal{L}_{S R 3}^{v}\left(L_{\text {Reputation }}\right)\left(\operatorname{see} \operatorname{RAND}\left(\mathcal{N} e i g_{v}, \mathcal{L}_{S R 3}^{v}\left(L_{\text {Reputation }}\right)\right)\right.$ in Lines 4 and 7$)$.

Informally, when a node needs to route a message, it draws at random a value from $L_{\text {Reputation }}$ plus a blank element. If the blank element is drawn, it selects a neighbor uniformly at random, and sends the message to that neighbor. Otherwise, 
$L_{\text {Reputation }}=[w, z, z], s_{R}=3$ for node $v$

Next hop probabilities for $v$ :

$$
\begin{aligned}
& P(X=w)=\frac{1+1 / 3}{4}=\frac{4}{12} \approx 33.33 \% \\
& P(X=z)=\frac{2+1 / 3}{4}=\frac{7}{12} \approx 58.33 \% \\
& P(X=y)=\frac{0+1 / 3}{4}=\frac{1}{12} \approx 8.33 \%
\end{aligned}
$$

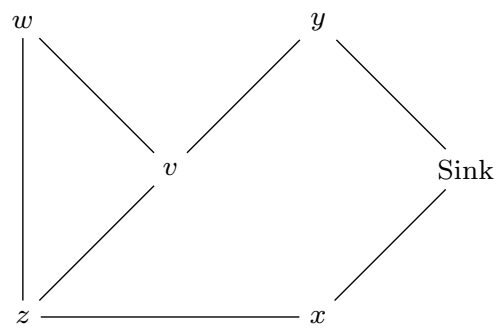

Fig. 1 An example of how the reputation affects the routing process

the message is sent to the neighbor whose identifier has been drawn. This way, the more a neighbor is trusted, the more it will be selected. However, because of the blank element, there is always a positive probability of selecting a neighbor without taking trust into account. Note that, initially $L_{\text {Reputation }}$ is empty, and consequently the first selections are made uniformly at random.

To ensure a better resiliency against attackers that change their behavior over time, and to reduce memory consumption, $L_{\text {Reputation }}$ is defined as a FIFO list of maximum size, $s_{R}$. The insertions in $L_{\text {Reputation }}$ use the operator $\bullet$ that satisfies the following condition: when the list is full, the next insertion is preceded by the removing of the oldest (and consequently, less relevant) element.

An example illustrating the probability law is provided in Figure 1. In this example, we focus on the node $v$ and assume a $L_{\text {Reputation }}$ of at most 3 elements. From the given configuration, $v$ will route most messages through $z$, because it has the greatest number of occurrences in the list $L_{\text {Reputation }}$ of $v$. The high reputation of $z$ may come from the fact that at some point the paths from $x$ to the sink through $z$ were faster and more reliable: this is a side effect of our protocol. Such fast and reliable paths indirectly help increasing the reputation of $z$, since in this case $x$ receives valid acknowledgments from $z$ more frequently than from other neighbors.

Using such a FIFO finite list, a node only stores the freshest information. Interestingly, if a compromised node first behaves well, its reputation increases, resulting in attracting the traffic. Then, it may change its behavior to become a blackhole (a node dropping all messages it receives). Now, thanks to our mechanism, regularly some messages will be routed via other nodes and consequently the reputation of the compromised node will gradually decrease, inducting then a severe reduction of the traffic going through that node.

Consider again Figure 1. If $z$ turns out to be compromised and starts dropping all messages, then all messages going through $z$ and $w$ will either get lost or loop back to $v$. However, there is still a positive probability that $v$ routes messages through $y$, which will retransmit them. Some of these messages will be delivered and consequently acknowledged. So, the identifiers currently stored in $L_{\text {Reputation }}$ will be progressively replaced by occurrences of $y$, increasing its probability (resp. decreasing the probability of $w$ and $z$ ) of being chosen. 


\subsection{Acknowledgment Routing}

An acknowledgment message ack is emitted because the corresponding data message $m$ has been successfully delivered by the sink. So, we can suppose that the path followed by $m$ was safe. Thus, we can use the bidirectionality of the links to route ack (as much as possible) through the reverse path followed by $m$.

This reverse routing is accomplished by letting a trail along the path followed by $m$. This trail is actually made using hash $\mathrm{H}\left(N_{v}\right)$ of the nonce $N_{v}$ identifying the message (see Line 2), this hash being stored in plaintext in the data message (see Line 6). The trail is then is stored thanks to the list $L_{\text {AckRouting }}$ maintained at each node: after the reception of each data message, the relaying nodes store the hash of the nonce available in the message, together with the identifier of the neighbor from which they received the message (Lines 14-17). This information will be then used during the return trip of the acknowledgment: when a node $v$ receives an acknowledgment containing the nonce $N_{x}$, it checks whether it is the final destination of that acknowledgment (Lines 20 and 25). If this is not the case, $v$ checks if an entry containing $\mathrm{H}\left(N_{x}\right)$ exists in $L_{\text {AckRouting }}$ (Lines 26-30). If $v$ finds such an entry, it sends the acknowledgment to the corresponding neighbor and removes the entry from $L_{\text {AckRouting }}$ (Line 29). Otherwise, the next hop of the acknowledgment is chosen uniformly at random, in a best-effort mindset $\left(\mathcal{L}_{R W}^{v}\right.$ denotes the probability law of the uniform random walk, see Line 31).

If a data message loops back to a node it already visited, the most relevant information regarding acknowledgments for this node is the oldest one. Therefore, before inserting a new trail, the node checks if $L_{\text {AckRouting }}$ already contains a trail for that message. If a related entry exists, we do not update $L_{\text {AckRouting }}$ (Lines 14-17).

Acknowledgments can be still dropped by compromised nodes. The trail for such lost acknowledgments would unnecessarily clutter the memory of nodes. To avoid this, we manage $L_{\text {AckRouting }}$ similarly to $L_{\text {Reputation }}$, i.e., $L_{\text {AckRouting }}$ is a list of bounded size $s_{A}$, appended using operator $\bullet$.

Finally, an intruder may build acknowledgments with false nonces. These fake acknowledgments will increase the load of the network, and impact the energy consumption. Now, some nodes being compromised, a safe node cannot trust information coming from its neighbors to decide whether it should forward or drop an acknowledgment. To circumvent that problem, a relay node decides to drop a received acknowledgment with probability $\frac{1}{N}$, where $N$ is an upper bound on the number of nodes (Lines 33 and 42). So, on the average, an acknowledgment makes $N$ hops in the network before being dropped. An interesting side effect of this method is the following: in a safe network (i.e., a network without attackers), the acknowledgments that follow long routes are often dropped before reaching their final destination. Since the length of the routes followed by the acknowledgments are directly related to the length of the route taken by the corresponding messages, the reputation mechanism ends up favoring shorter routes, thus improving the overall hops complexity.

The overall behavior of SR3 is summarized in Figure 2. 


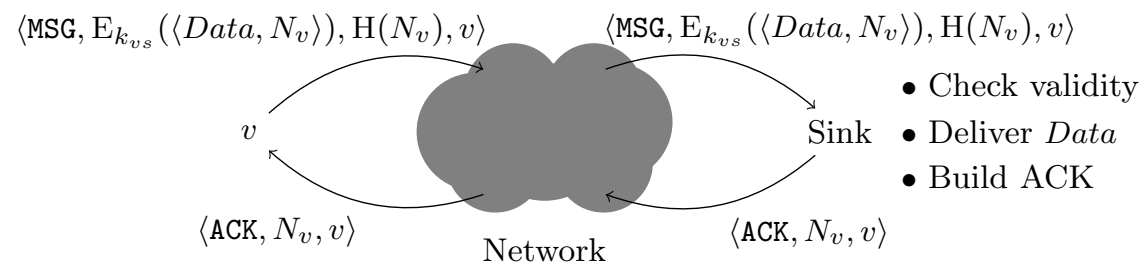

Fig. 2 A message and its acknowledgment.

\section{Security Analysis}

We evaluate the security of SR3 in two phases. The first phase focuses on the message format, for which we prove the following three properties: unforgeability, confidentiality of the data, and confidentiality of the nonce before message delivery. This analysis uses the tool Crypto Verif [12]. We first detail the modeling of SR3 and our intruder model. Then, we model the different security properties we considered. CryptoVerif automatically finds bounds on the security of these properties. We refined these bounds to allow the user to determine the desired trade-off between message sizes and the expected security level. We illustrate our results with an example based on the classical data link protocol $S-M A C[35]^{3}$ which is dedicated to WSNs and allows to transmit up to 250 bytes of data in a message.

The second phase is a symbolic analysis of the protocol, in order to prove its security when running several sessions. This analysis supposes that the cryptographic primitives are perfect. For this, we use the tool Scyther [15].

We first describe how we model SR3 and the attacker for these two analyses.

\subsection{Modeling of SR3}

SR3, as described in the previous section, routes messages through several nodes. There are three distinct roles in this process: the source (whose identifier is denoted by $s r c$ ) of the considered data message, the relays, and the sink.

The data message is initially created by the source, which then forwards it either to a relay or the sink. Initially, a data message consists of a ciphertext $C=\mathrm{E}_{k_{s r c}}(\langle$ Data, $N\rangle)$ containing the (symmetric) authenticated encryption of some data Data and a nonce $N$, and a plaintext part made of the hash of the nonce $N$ and the identifier src. Notice that the symmetric key $k_{s r c}$ is chosen uniformly at random in $\mathcal{K}$, the space of all the possible symmetric keys, before the WSN is deployed. We suppose $k_{s r c}$ is known only by the source src and the sink.

Relays forward the data message without changing it. When a data message reaches the sink, this latter first checks whether the message respects the format $\langle$ MSG $, C, h, s\rangle$. If so, $C$ is decrypted with the key of the node identified by $s$ $\left(\mathrm{E}_{k e y s[s]}^{-1}(C)\right)$ : if this operation succeeds, then a valid data is extracted and delivered, moreover an acknowledgment $\langle\mathrm{ACK}, N, s r c\rangle$, is generated and routed through relays until reaching the source.

\footnotetext{
3 S-MAC stands for Sensor Medium Access Control.
} 


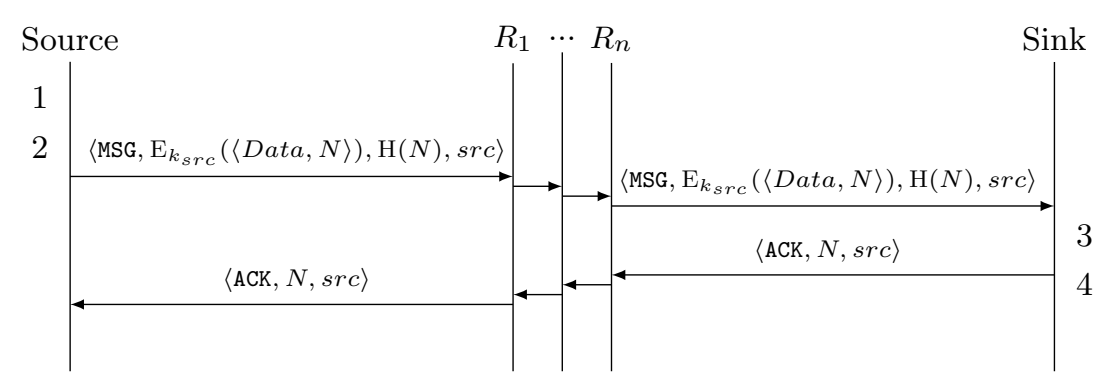

1. Generate Data, 2. Draw a nonce $N$,

3. Check validity, 4. Deliver Data

Fig. 3 One session of the SR3 protocol

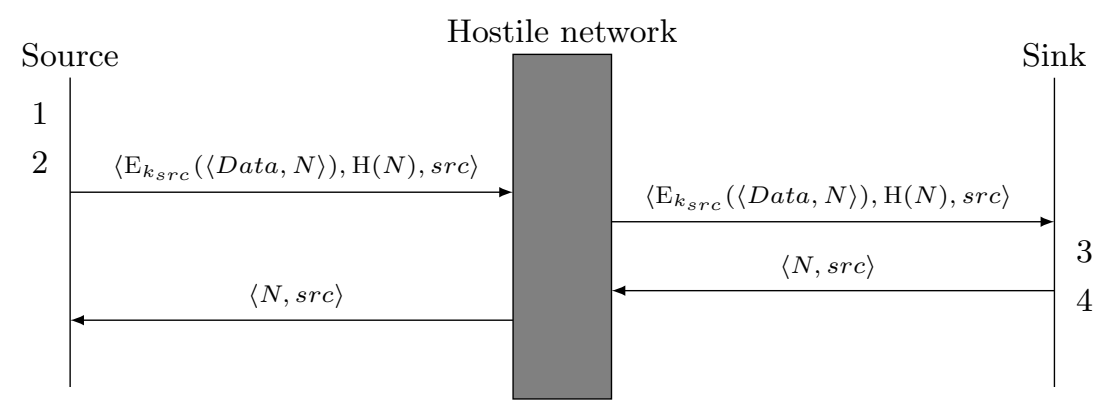

1. Generate Data, 2. Draw a nonce $N$,

3. Check validity, 4. Deliver Data

Fig. 4 Modeling of one session of SR3

A data message can loop back to its source, and an acknowledgment can loop back to the sink: they act as relays in these cases.

Figure 3 illustrates that view of the protocol. The honest relays do not alter the messages in any way (however, remind that honest nodes may sometimes drop ACK messages), and the protocol works with any number of them, or none at all. Also, our intruder model specifies that any node can be compromised, except the sink. Therefore, all relays $R_{i}$ are suspicious, and we lump them, whether honest or compromised, together in one single entity, called the hostile network. All communications between the source and the sink happen through the hostile network, as depicted in Figure 4. The hostile network can modify or drop messages, and can also create messages using informations deduced from previous communications. (As the types MSG and ACK can be deduced from the message format, we omit them in the modeling.) 
3.2 Game-Based Proof of the Security Properties in the Computational Model

We now consider the message format.

\subsubsection{Background}

We model the ability of our protocol to meet a given security property using games. A game is a probabilistic algorithm where an adversary, given as a probabilistic polynomial-time Turing machine, faces a challenge, which consists in breaking a specific property modeled by the game. The goal is then to compute the ability of the adversary to win the challenge. This is called the advantage of the adversary.

When describing a game, we write $a \stackrel{\$}{\leftarrow} X$ to denote that $a$ is a random value obtained according the distribution represented by $X$. If $X$ is a set, $a$ is drawn uniformly at random on $X$. Similarly, if $X$ is a probabilistic algorithm, $a$ is drawn randomly using the algorithm.

Our analysis uses a common cryptography model, called the random oracle model [10], where hash functions are modeled by random oracles. A random oracle is a theoretical black box $\mathcal{O}$ which satisfies the following property: for every input $i$, the first time $\mathcal{O}$ is queried with $i, \mathcal{O}$ returns a value $v$, picked uniformly at random from its output domain; then, each time $\mathcal{O}$ is queried again with $i, \mathcal{O}$ returns the same value $v$. Moreover, encryptions are modeled using encryption oracles. An encryption oracle is a theoretical black box which retains the secret encryption key and encrypts arbitrary data at the adversary's request.

\subsubsection{Modeling SR3's Primitives}

Hash function. Our algorithm uses a hash function of input size $\eta_{n}$ and of output size $\eta_{h}$. We model it as a random oracle, and we refer to this modeling using $\mathcal{H}:\{0,1\}^{\eta_{n}} \rightarrow\{0,1\}^{\eta_{h}}$.

Nonces. Nonces are modeled as truly random numbers of size $\eta_{n}$.

Encryption scheme. We assume the authenticated encryption is both IND-CCA2 (INDistinguishability against adaptive Chosen-Ciphertext Attack) [32] and INT-PTXT [11,25] (INTegrity of the PlainTeXTs).

Intuitively, an encryption primitive is $I N D-C C A 2$, if it is computationally difficult for an adversary to win a challenge that consists in guessing which of two data is encrypted into a given ciphertext despite it has access to a decryption and an encryption oracle before and after the reception of the challenge. More formally, 
we recall below the IND-CCA2 game introduced in [9].

$$
\begin{aligned}
\text { Experiment } & \text { Expt } \\
& K \stackrel{\$}{I N D-C C A 2}(\mathcal{K}): \\
& \left(D_{0}, D_{1}, \text { state }\right) \stackrel{\$}{\leftarrow} \mathcal{A}_{1}^{\mathcal{E}(K, \cdot), \mathcal{E}^{-1}(K, \cdot)}() \\
& b \stackrel{\$}{\leftarrow}\{0,1\} \\
& C \stackrel{\$}{\leftarrow} \mathcal{E}\left(K, D_{b}\right) \\
& \text { Return } b=\mathcal{A}_{2}^{\mathcal{E}(K, \cdot), \mathcal{E}^{-1}(K, \cdot)}\left(D_{0}, D_{1}, C, \text { state }\right)
\end{aligned}
$$

First, a key $K$ is generated uniformly at random from $\mathcal{K}$, the set of possible (symmetric) keys, of size $\eta_{k}$. Then, the adversary $\mathcal{A}$ runs in two phases: $\mathcal{A}_{1}$ and $\mathcal{A}_{2}$. In each of them, the encryption and decryption oracles, respectively denoted by $\mathcal{E}(K, \cdot)$ and $\mathcal{E}^{-1}(K, \cdot)$, can be called a polynomial number of times. $\mathcal{A}_{1}$ outputs two distinct data $D_{0}$ and $D_{1}$ of identical size and some information state used to link the two attacker phases together. Then, one of the data $D_{0}$ and $D_{1}$ is selected uniformly at random and encrypted in the ciphertext $C$. Finally, $\mathcal{A}_{2}$ receives the challenge (i.e., the two data $D_{0}, D_{1}$, as well as the ciphertext $C$ ) and state. The adversary $\mathcal{A}_{2}$ is allowed to call $\mathcal{E}^{-1}(K, \cdot)$ with any ciphertext, except the challenge ciphertext $C$. The game returns 1 if and only if the adversary correctly guesses the value of the challenge bit $b$, the index of the data encrypted into $C$. For any adversary $\mathcal{A}$, the $I N D-C C A 2$ advantage in this game, noted $\operatorname{Adv}^{I N D-C C A 2}(\mathcal{A})$, is defined as:

$$
\operatorname{Adv}^{I N D-C C A 2}(\mathcal{A})=2 \times \operatorname{Pr}\left[\mathbf{E x p t}^{I N D-C C A 2}(\mathcal{A})=1\right]-1
$$

where $\operatorname{Pr}\left[\operatorname{Expt}^{I N D-C C A 2}(\mathcal{A})=1\right]$ is the probability that $\mathcal{A}$ wins the IND-CCA2 game.

Intuitively, an encryption primitive $E$ is INT-PTXT, if it is computationally difficult for an adversary to produce a valid ciphertext decrypting to a data which had never been encrypted using $E$. Below, we recall the game used in [9] to define this notion for a symmetric encryption scheme $(\mathcal{K}, E, D)$, where $\mathcal{K}, E$, and $D$ are respectively a set of keys (of size $\eta_{k}$ ), and encryption and decryption primitives.

$$
\begin{aligned}
& \text { Experiment } \operatorname{Expt}^{I N T-P T X T}(\mathcal{A}): \\
& K \stackrel{\$}{\leftarrow} \mathcal{K} \\
& S \leftarrow \emptyset ; \\
& C \stackrel{\$}{\leftarrow} \mathcal{A}^{\mathcal{E}_{S}(K, \cdot), \mathcal{E}^{-1}(K, \cdot)}() \\
& d \leftarrow \mathcal{D}(K, C) \\
& \text { Return } d \neq \perp \text { and } d \notin S
\end{aligned}
$$

Each call to the encryption oracle $\mathcal{E}_{S}(K, d)$ consists of storing the data $d$ into $S$, and then returning $E(K, d)$, the encryption of $d$ using key $K$. The adversary also has access to the decryption oracle $\mathcal{E}^{-1}(K, \cdot)$. The adversary $\mathcal{A}$ wins the INTPTXT game if and only if it can forge a valid ciphertext $C$ whose corresponding 
decryption has never been queried to the encryption oracle. For any adversary $\mathcal{A}$, the INT-PTXT advantage in this game, noted $\mathbf{A d v}^{I N T-P T X T}(\mathcal{A})$, is defined as:

$$
\mathbf{A d v}^{I N T-P T X T}(\mathcal{A})=\operatorname{Pr}\left[\mathbf{E x p t}{ }^{I N T-P T X T}(\mathcal{A})=1\right]
$$

where $\operatorname{Pr}\left[\operatorname{Expt}^{I N T-P T X T}(\mathcal{A})=1\right]$ is the probability that $\mathcal{A}$ wins the INT-PTXT game.

Since we assume the encryption scheme of SR3 is both IND-CCA2 and INTPTXT-secure, both $\mathbf{A d v}^{I N D-C C A 2}(\mathcal{A})$ and $\mathbf{A d v}^{I N T-P T X T}(\mathcal{A})$ become negligible in the size of the keys of encryption scheme $\eta_{k}$. An advantage $\operatorname{Adv}(\mathcal{A})$ becomes negligible in $x$, if for every positive polynomial $P(x)$, we have $\exists K, \forall x>K, \operatorname{Adv}(\mathcal{A})<\frac{1}{P(x)}$.

\subsubsection{Modeling SR3}

Based on the above presented model, we describe actions performed by the source and sink using two functions, see Figure 5. The attacker has access to some of these functions. For instance, the function $\operatorname{Gen}^{\mathrm{E}_{k_{s r c}}(\cdot)}(\cdot)$ below represents the normal behavior of a node, and an attacker who has access to this function models a chosen-plaintext attack.

- Gen ${ }^{\mathrm{E}_{k_{s r c}}(\cdot)}($ Data $)$ is the function which generates a message produced by src containing Data (whose length is $\eta_{d}$ ), using the encryption function from $\{0,1\}^{\eta_{p}}$ to $\{0,1\}^{\eta_{c}}$ (where $\eta_{p}=\eta_{d}+\eta_{n}$ and $\eta_{c} \geq \eta_{p}$ ). This message is made of $\langle C, h, s r c\rangle=\left\langle\mathrm{E}_{k_{s r c}}(\langle\right.$ Data, $\left.N\rangle), \mathrm{H}(N), s r c\right\rangle$, where $N$ is a fresh unpredictable nonce of size $\eta_{n}, \mathrm{H}(N)$ is the hash of $N$, and $\mathrm{E}_{k_{s r c}}(\langle$ Data, $N\rangle)$ is the encryption of the concatenation of the data and nonce. The function $G_{e n}{ }^{\mathrm{E}_{k_{s r c}}(\cdot)}($ Data) returns the pair $\langle C, h, s r c\rangle, N$. The nonce $N$ is given in cleartext to represent the knowledge of an attacker that listens to traffic in the network. Indeed, such an attacker may have access to both the messages and their acknowledgments; he may then know the nonces contained in the original messages.

We store all the encrypted ciphertexts in a set called Queries, initially empty.

- Verif $\mathrm{E}_{k_{s r c}}^{-1}(\cdot)(\langle C, h, s\rangle)$ is the function that checks whether the packet $\langle C, h, s\rangle$ is valid or not. Precisely, it checks

- whether $s=s r c$,

- whether $\mathrm{E}_{k_{s c}}^{-1}(C) \neq \perp$, which guarantees the integrity of the encrypted data (e.g., $\mathrm{E}_{k_{s r c}}^{-1}(C)$ fails if some digit has been reversed), and authenticity (since encryption uses a symmetric key, only entities knowing this key, i.e., the source and sink, are able to correctly encrypt and decrypt data, consequently integrity implies authenticity), and

- whether $h=\mathrm{H}(N)$, where $\langle d, N\rangle=\mathrm{E}_{k_{s r c}}^{-1}(C)$ (of course, this last check is made only if the two first ones succeed).

If these three conditions are satisfied, then the function outputs 1 (meaning that the message is valid), 0 otherwise.

These two functions allow us to model SR3 in CryptoVerif. Notice that, in the following games, we replace $\mathrm{E}_{k_{s r c}}(\cdot), \mathrm{E}_{k_{r c}}^{-1}(\cdot)$, and $\mathrm{H}(\cdot)$ - the encryption, decryption, and hash functions actually used by SR3 - by the encryption, decryption, and random oracles $\mathcal{E}\left(k_{s r c}, \cdot\right), \mathcal{E}^{-1}\left(k_{s r c}, \cdot\right)$, and $\mathcal{H}(\cdot)$ that respectively model them. These oracles represent knowledge accessible to the intruder. 


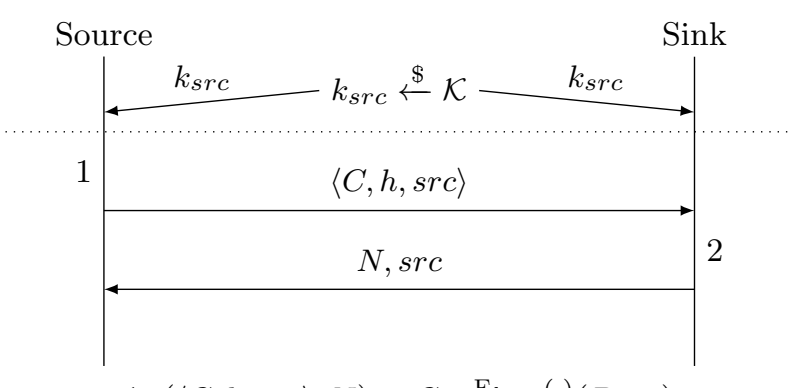

1. $(\langle C, h, s r c\rangle, N)=G_{e n} \mathrm{E}_{k_{s r c}(\cdot)}($ Data $)$

2. Message verification phase: $\operatorname{Verif}^{\mathrm{E}_{k_{s r c}}^{-1}(\cdot)}(C)$

Fig. 5 The SR3 protocol, using functions

\subsection{Properties}

The three properties we want to prove are the following:

- Confidentiality of the (encrypted) data: the probability of the adversary getting information about the data in a message is negligible, even when the acknowledgment has been sent.

- Confidentiality of the nonce: the probability of the adversary getting information about the nonce $N$ in a message, before the message has been delivered, is negligible.

- Unforgeability: the probability that the adversary creates a new ciphertext $C$ such that $\operatorname{Verif} \mathrm{E}_{k_{s r c}}^{-1}(\cdot)(C)=1$ is negligible.

With the help of CryptoVerif, we analyzed those three properties of SR3. Each of these three properties is evaluated thanks to a game. For each game, CryptoVerif outputs a bound on the advantage of any adversary in that game. This bound is obtained automatically after successive game reductions. The complete verification code is available online [4].

\subsubsection{Data Confidentiality}

The first property we consider is the confidentiality of the data. The game (named FG, for Find-then-Guess) is based on the idea that even if the adversary chooses the set of possible data, it cannot guess which of those data is inside a given message. On the other hand, if the attacker was able to win reliably, it would be also effectively able to recover some information about the data contained in messages, without knowledge of the key.

Let $\mathcal{A}$ be an adversary running in two phases: $\mathcal{A}_{1}$ and $\mathcal{A}_{2}$. First, $\mathcal{A}_{1}$ outputs two data, $D_{0}$ and $D_{1}$, of identical size together with some information state used to link the two attacker phases together. One of these two data is selected uniformly at random, and a data message $\langle C, h, s r c\rangle$ is generated using the selected data and the key $k_{s r c}$ of $s r c$, initially generated uniformly at random from $\mathcal{K}$. Then, $\langle C, h, s r c\rangle$, the nonce $N$ it contains, and state are given to $\mathcal{A}_{2}$. To win, $\mathcal{A}_{2}$ should guess which of the two data is in $C$. During this game, $\mathcal{A}$ can query $\operatorname{Gen}^{\mathcal{E}\left(k_{s r c}, \cdot\right)}(\cdot)$, 


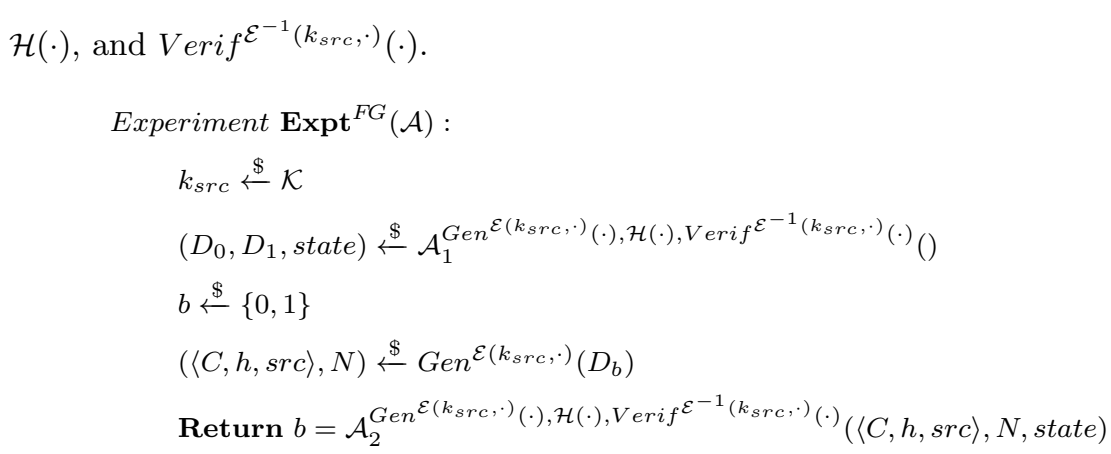

Let $\operatorname{Pr}\left[\operatorname{Expt}^{F G}(\mathcal{A})=1\right]$ be the probability of winning the find-then-guess game. We define the find-then-guess advantage of $\mathcal{A}$ against $F G$, noted $\operatorname{Adv}^{F G}(\mathcal{A})$, as follows:

$$
\operatorname{Adv}^{F G}(\mathcal{A})=2 \times \operatorname{Pr}\left[\mathbf{E x p t}^{F G}(\mathcal{A})=1\right]-1
$$

We modeled this game in CryptoVerif to obtain a bound on $\operatorname{Adv}^{F G}(\mathcal{A})$. Actually, this bound depends on the ability of the attacker $\mathcal{A}$ to break the encryption scheme used by SR3, i.e., this game can be reduced to the IND-CCA2 game. This means that the bound on $\operatorname{Adv}^{F G}(\mathcal{A})$ depends on the advantage $\operatorname{Adv}^{I N D-C C A 2}(\mathcal{B})$ of some adversary $\mathcal{B}$ in the IND-CCA2 game. Precisely, for all adversaries $\mathcal{A}$

- making $q_{G}$ queries to $G e n^{\mathcal{E}\left(k_{s r c}, \cdot\right)}(\cdot), q_{V}$ queries to $\operatorname{Verif} \mathcal{E}^{-1}\left(k_{s r c}, \cdot\right)(\cdot)$, and $q_{H}$ queries to $\mathcal{H}(\cdot)$ in the $F G$ game, and

- running the $F G$-game in $T_{\mathcal{A}}$ time units,

there exists an adversary $\mathcal{B}$

- making $q_{G}+1$ queries to the encryption oracle $\mathcal{E}\left(k_{s r c}, \cdot\right)$ and $q_{V}$ queries to the decryption oracle $\mathcal{E}^{-1}\left(k_{s r c}, \cdot\right)$ in the IND-CCA2 game, and

- running the $I N D-C C A 2$ game in $T_{\mathcal{B}}$ time units with $T_{\mathcal{B}}=T_{\mathcal{A}}+P_{1}\left(q_{G}, q_{V}, s\right)$ time units, where $P_{1}\left(q_{G}, q_{V}, s\right)$ is polynomial in $q_{G}, q_{V}$, and the message size $s$ (see [4] for details)

such that

$$
\operatorname{Adv}^{F G}(\mathcal{A}) \leq 2 \times \operatorname{Adv}^{I N D-C C A 2}(\mathcal{B})
$$

Note that the IND-CCA2 property of the encryption scheme allows to obtain a security bound which is independent from $\eta_{c}$. Also, $q_{H}$ does not appear in the result meaning that calls to $\mathcal{H}(\cdot)$ does not help the adversary in anyway.

Moreover, IND-CCA2 guarantees that for all adversaries $\mathcal{B}, \mathbf{A d v}^{I N D-C C A 2}(\mathcal{B})$, and consequently $\operatorname{Adv}^{F G}(\mathcal{A})$, becomes negligible in $\eta_{k}$, the size of the key. We now refine and instantiate this bound to select the necessary trade-off between the desired level of security and the mandatory minimization of the message overhead.

Illustrative Example. As an example, we now show how to fix parameters when focusing on the $S-M A C$ protocol [35] for which the data packet length can be up to 250 bytes. 
Recall that we want to achieve the IND-CCA2 and INT-PTXT-secure properties. Such properties can be ensured using an Encrypt-then-MAC primitive [9]. Encryptthen-MAC consists of an encryption primitive, e.g., AES-128 [13], ${ }^{4}$ and a Message Authentication Code (MAC) [7], e.g., HMAC-SHA-1 or HMAC-SHA-256. ${ }^{5}$

Here, we use AES-128 [13], which outputs 128 bits (16 bytes) of ciphertext using an input plaintext of 128 bits. To overcome the size limitation, we use AES-128 together with a block cipher mode of operation called Cipher Block Chaining $(C B C)$ [17]. This latter allows to securely link together several fixed-length ciphertexts, so-called blocks. Using AES, each block will be of size 128 bits (16 bytes), and the overhead of CBC is one block (the initialization vector), so 16 bytes. We fix here the size of the plaintext to 208 bytes, i.e., 13 blocs of 16 bytes. So, using AES-128 together with $\mathrm{CBC}$, we obtain a ciphertext constituted of 14 blocs of 16 bytes, i.e., 224 bytes.

Then, for the MAC, we use HMAC-SHA-256 which, given an input of up to $2^{64}$ bits, produces an output message authentication code (MAC) of 256 bits (32 bytes). However to reduce the overhead, we truncate this output to 9 bytes, i.e. 72 bits. Overall, we obtain a ciphertext of 233 bytes.

Then, using these parameters and several results from $[5,6,8,9]$, we obtain the following bound (see [4] for details):

$$
\begin{aligned}
\mathbf{A d v}^{F G}(\mathcal{A}) \leq & 4 \times \mathbf{A d v}_{\text {Comp-SHA-256* }}^{R K A}(\mathcal{M})+4 \times \operatorname{Adv}_{\text {Comp-SHA-256 }}^{P R F}(\mathcal{N})+ \\
& 2 \times\left(q_{V}-1\right) \times q_{V} \times\left(8 \times \mathbf{A d v}_{\text {Comp-SHA-256 }}^{P R F}(\mathcal{O})+\frac{1}{2^{256}}\right)+ \\
& \frac{q_{V}}{2^{70}}+4 \times \operatorname{Adv}_{\mathrm{AES}}^{P R F}(\mathcal{I})+\frac{196 \times\left(q_{G}+1\right)^{2}}{2^{127}}
\end{aligned}
$$

where

- comp-SHA-256 (resp. comp-SHA-256*) is the compression function of SHA-256 (resp. its dual function);

- the PRF-advantage $\operatorname{Adv}_{F}^{P R F}(\mathcal{X})$ measures the ability of an adversary $\mathcal{X}$ to guess whether a given oracle is a random instance of $F$, a family of pseudorandom functions, or a truly random function;

- $\operatorname{Adv}_{F}^{R K A}(\mathcal{X})$ is the PRF-advantage of $\mathcal{X}$ under related key attacks;

- I runs in time $O\left(T_{\mathcal{B}}\right)$ and makes $14 \times\left(q_{G}+1\right)$ queries to the encryption oracle, modeling the encryption function of AES;

$-\mathcal{M}$ is a related key adversary that performs two oracle queries and has time $O\left(T_{\mathcal{B}}\right)$

$-\mathcal{N}$ makes $q_{V}$ queries and runs in $O\left(T_{\mathcal{B}}\right)$ time;

- $\mathcal{O}$ makes 2 queries and runs in $O(T), T$ being the time for one computation of comp-SHA-256.

We assume $q_{V}=2^{20}$ and $q_{G}=2^{30}$. Finally, we bound the strength of the adversaries using estimations based on the current best attacks on AES $\left(2^{126.1}\right)$ and comp-SHA-256 $\left(2^{256}\right)$ :

- For AES, if the attacker can make $N_{\mathrm{AES}}$ queries, its advantage can be estimated by $\frac{N_{\mathrm{AES}}}{2^{126.1}}$.

\footnotetext{
4 AES stands for Advanced Encryption Standard.

${ }^{5}$ HMAC-SHA stands for Keyed-Hash Message Authentication Code and Secure Hash Algorithm.
} 
- For SHA-256, if the attacker can make $N_{\mathrm{SHA}}$ queries to the compression function, then the advantage of the attacker can be estimated by $\frac{N_{\mathrm{SH}}}{2^{256}}$.

We assume $N_{\mathrm{AES}} \leq 2^{70}$ and $N_{\mathrm{SHA}} \leq 2^{100}$. Hence, we obtain $\operatorname{Adv}^{F G}(\mathcal{A}) \leq 2^{-49}$.

\subsubsection{Nonce Confidentiality}

In the next game, we evaluate whether an adversary can extract a nonce from an undelivered message. Let $\mathcal{A}$ be an adversary running in two phases $\left(\mathcal{A}_{1}\right.$ and $\left.\mathcal{A}_{2}\right)$ that communicate using a variable named state. The game consists in giving a challenge data message $\langle C, h, s r c\rangle$ to an adversary $\mathcal{A}$, who should guess the nonce inside this message in at most $n b_{A}$ tries. To do this, the adversary is allowed to call $\operatorname{Gen}^{\mathcal{E}\left(k_{s r c}, \cdot\right)}(\cdot), \mathcal{H}(\cdot)$, and $\operatorname{Verif}^{\mathcal{E}^{-1}\left(k_{s r c}, \cdot\right)}(\cdot)$. Moreover, it chooses the data that will be contained in the challenge message.

$$
\begin{aligned}
& \text { Experiment } \operatorname{Expt}^{N-\operatorname{conf}}(\mathcal{A}) \text { : } \\
& k_{\text {src }} \stackrel{\$}{\leftarrow} \mathcal{K} \\
& (\text { Data, state }) \stackrel{\$}{\leftarrow} \mathcal{A}_{1}^{\operatorname{Gen} \mathcal{E}^{\mathcal{E}\left(k_{s r c}, \cdot\right)}(\cdot), \mathcal{H}(\cdot), \text { Verif }^{\mathcal{E}^{-1}\left(k_{s r c}, \cdot\right)}(\cdot)}() \\
& (\langle C, h, s r c\rangle, N) \stackrel{\$}{\leftarrow} \operatorname{Gen}^{\mathcal{E}\left(k_{s r c}, \cdot\right)}(\text { Data })
\end{aligned}
$$

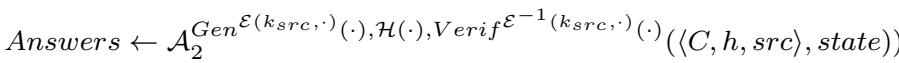

$$
\begin{aligned}
& \text { Return }\left(\mid \text { Answers } \mid \leq n b_{A} \wedge N \in \text { Answers }\right)
\end{aligned}
$$

The nonce confidentiality advantage of $\mathcal{A}$ against $N$-conf is defined as the probability of winning the game, i.e., $\operatorname{Pr}\left[\operatorname{Expt}^{N-\operatorname{conf}}(\mathcal{A})=1\right]$ :

$$
\operatorname{Adv}^{N-\operatorname{conf}}(\mathcal{A})=\operatorname{Pr}\left[\operatorname{Expt}^{N-\operatorname{conf}}(\mathcal{A})=1\right]
$$

CryptoVerif outputs that for all adversaries $\mathcal{A}$ :

- making $q_{G}$ queries to $G e n^{\mathcal{E}\left(k_{s r c}, \cdot\right)}(\cdot), q_{V}$ queries to $\operatorname{Verif} \mathcal{E}^{-1}\left(k_{s r c} \cdot \cdot\right)(\cdot), q_{H}$ queries to $\mathcal{H}(\cdot)$, and $n b_{A}$ tries in the $N-$ conf game, and

- running the $N$-conf game in $T_{\mathcal{A}}$ times units,

there exists an adversary $\mathcal{B}$ :

- making $q_{G}+1$ queries to $\mathcal{E}\left(k_{s r c}, \cdot\right)$ and $q_{V}$ queries to $\mathcal{E}^{-1}\left(k_{s r c}, \cdot\right)$ in the IND$C C A 2$ game, and

- running the IND-CCA2 game in $T_{\mathcal{B}}$ time units with $T_{\mathcal{B}}=T_{\mathcal{A}}+P_{2}\left(q_{G}, q_{V}, s\right)$ time units, where $P_{2}\left(q_{G}, q_{V}, s\right)$ is polynomial in $q_{G}, q_{V}$, and the message size $s$ (see [4] for details),

such that:

$$
\operatorname{Adv}^{N-\operatorname{conf}}(\mathcal{A}) \leq \frac{n b_{A}+q_{H}+q_{G}}{2^{\eta_{n}}}+\operatorname{Adv}^{I N D-C C A 2}(\mathcal{B})
$$

Similarly to the previous property, this bound becomes negligible when increasing $\eta_{n}$ and $\eta_{k}$. Again, one can find the right values to obtain a given security bound. 
Illustrative Example. Considering the encryption parameter values already fixed in Subsections 3.3.1 and the results from $[5,6,8,9]$, we obtain the following bound (see [4] for details):

$$
\begin{aligned}
\operatorname{Adv}^{N-\operatorname{conf}}(\mathcal{A}) \leq & \frac{n b_{A}+q_{H}+q_{G}}{2^{\eta_{n}}}+2 \times \mathbf{A d v}_{\text {Comp-SHA-256* }}^{R K A}(\mathcal{M})+ \\
& 2 \times \mathbf{A d v}_{\mathrm{Comp}-\mathrm{SHA}-256}^{P R F}(\mathcal{N})+ \\
& \left(q_{V}-1\right) \times q_{V} \times\left(8 \times \mathbf{A d v}_{\mathrm{Comp}-\mathrm{SHA}-256}^{P R F}(\mathcal{O})+\frac{1}{2^{256}}\right)+ \\
& \frac{q_{V}}{2^{71}}+2 \times \operatorname{Adv}_{\mathrm{AES}}^{P R F}(\mathcal{I})+\frac{196 \times\left(q_{G}+1\right)^{2}}{2^{128}}
\end{aligned}
$$

$(\mathcal{I}, \mathcal{M}, \mathcal{N}, \mathcal{O}$ are the same adversaries as those defined in Subsection 3.3.1.)

To obtain an estimation, we use the same values as in Subsection 3.3.1 and we assume $n b_{A}=q_{V}=2^{20}$ and $q_{H}=2^{40}$. Finally, we fix the size $\eta_{n}$ of the nonce to 96 (12 bytes). Hence, we obtain $\operatorname{Adv}^{N-\operatorname{conf}}(\mathcal{A}) \leq 2^{-49}$, using a ciphertext of length 233 bytes and a nonce of 12 bytes.

\subsubsection{Unforgeability}

Finally, the last game evaluates the unforgeability, i.e., the ability of an intruder to create a new valid ciphertext. Note that this property implies both indistinguishability and authenticity of the data. To evaluate unforgeability, the game gives an attacker $\mathcal{A}$ access to both $G e n^{\mathcal{E}\left(k_{s r c}, \cdot\right)}(\cdot)$ and $\operatorname{Verif}^{\mathcal{E}^{-1}\left(k_{s r c}, \cdot\right)}(\cdot)$. To win, $\mathcal{A}$ should return a ciphertext which is valid and which has never been encrypted by $G e n^{\mathcal{E}\left(k_{s r c}, \cdot\right)}(\cdot)$. Recall that the set Queries contains the ciphertext encrypted by $\operatorname{Gen}^{\mathcal{E}\left(k_{s r c}, \cdot\right)}(\cdot)$.

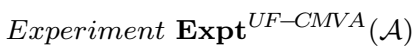

$$
\begin{aligned}
& \text { Queries } \leftarrow \emptyset \\
& k_{\text {src }} \stackrel{\$}{\leftarrow} \mathcal{K}
\end{aligned}
$$

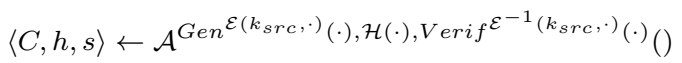

$$
\begin{aligned}
& \text { Return } C \notin \text { Queries } \wedge \text { Verif } \mathcal{E}^{-1}\left(k_{s r c}, \cdot\right)(C)
\end{aligned}
$$

The unforgeability advantage of $\mathcal{A}$ against $U F-C M V A$, noted $\mathbf{A d v}^{U F-C M V A}(\mathcal{A})$, is defined as the probability of $\mathcal{A}$ winning this game, $\operatorname{Pr}\left[\operatorname{Expt}^{U F-C M V A}(\mathcal{A})=1\right]$ :

$$
\mathbf{A d v}^{U F-C M V A}(\mathcal{A})=\operatorname{Pr}\left[\mathbf{E x p t} \mathbf{t}^{U F-C M V A}(\mathcal{A})=1\right]
$$

Using CryptoVerif, we find that the advantage of $\mathcal{A}$ depends only the strength of the authenticated encryption. Formally, for all adversaries $\mathcal{A}$ :

- making $q_{G}$ queries to $G e n^{\mathcal{E}\left(k_{s r c}, \cdot\right)}(\cdot), q_{V}$ queries to $\operatorname{Verif} \mathcal{E}^{-1}\left(k_{s r c}, \cdot\right)(\cdot), q_{H}$ queries to $\mathcal{H}(\cdot)$ in the $U F-C M V A$ game, and

- running the $U F-C M V A$ game in $T_{\mathcal{A}}$ time units,

there exists an adversary $\mathcal{B}$ :

- making $q_{G}$ queries to $\mathcal{E}\left(k_{s r c}, \cdot\right)$ and $q_{V}+1$ queries to $\mathcal{E}^{-1}\left(k_{s r c}, \cdot\right)$ in the INTPTXT game, and 
- running the INT-PTXT game in $T_{\mathcal{B}}$ time units with $T_{\mathcal{B}}=T_{\mathcal{A}}+P_{3}\left(q_{G}, q_{V}, s\right)$ time units, where $P_{3}\left(q_{G}, q_{V}, s\right)$ is polynomial in $q_{G}, q_{V}$, and the message size $s$ (see [4] for details)

such that:

$$
\mathbf{A d} \mathbf{v}^{U F-C M V A}(\mathcal{A}) \leq \mathbf{A d} \mathbf{v}^{I N T-P T X T}(\mathcal{B})
$$

Similarly to the previous properties, $\operatorname{Adv}^{I N T-P T X T}(\mathcal{B})$ becomes negligible when increasing $\eta_{k}$.

Illustrative Example. Considering the encryption parameter values already fixed in Subsections 3.3.1-3.3.2 and the results from [5,6,8,9], we obtain the following bound (see [4] for details):

$$
\begin{aligned}
\mathbf{A d v} \mathbf{v}^{U F-C M V A}(\mathcal{A}) \leq & \mathbf{A d} \mathbf{d v}_{\text {Comp-SHA-256* }}^{R K A}\left(\mathcal{M}^{\prime}\right)+\mathbf{A d v}_{\text {Comp-SHA-256 }}^{P R F}\left(\mathcal{N}^{\prime}\right)+ \\
& \frac{\left(q_{V}-1\right) \times q_{V}}{2} \times\left(8 \times \mathbf{A d v}_{\text {Comp-SHA-256 }}^{P R F}\left(\mathcal{O}^{\prime}\right)+\frac{1}{2^{256}}\right)+\frac{q_{V}}{2^{72}}
\end{aligned}
$$

where

- $\mathcal{M}^{\prime}$ is a related key adversary that performs two oracle queries and has time $O\left(T_{\mathcal{B}}\right)$

- $\mathcal{N}^{\prime}$ makes $q_{V}$ queries and runs in $O\left(T_{\mathcal{B}}\right)$ time; and

- $\mathcal{O}^{\prime}$ makes 2 queries and runs in $O(T), T$ being the time for one computation of comp-SHA-256.

To obtain an estimation, we use the same values as in Subsections 3.3.1-3.3.2. We obtain $\mathbf{A d v} \mathbf{v}^{U F-C M V A}(\mathcal{A}) \leq 2^{-51}$.

Overall, we obtain a security level of at least $2^{-49}$ for each of the three aforementioned properties (unforgeability, confidentiality of the data, and confidentiality of the nonce before message delivery) using a ciphertext of length 233 bytes and a nonce of 12 bytes. As the nonce is concatenated to the data in the plaintext to encrypt (208 bytes), the size of the data is then at most 196 bytes. Note also that the size of the hash has to be fixed. Here, we fix it to 12 bytes (the same size as the nonce), to prevent random collisions. In addition to the ciphertext (233 bytes) and hash (12 bytes), a data message also contains the identifier of the source (4 bytes) and one bit for the message type (MSG). Hence, the data messages can be encoded using less than 250 bytes, with a low overhead as up to 196 bytes of data can be stored in, and so are supported by $S$-MAC. Similarly, our acknowledgment messages are supported by $S$-MAC since they can be encoded using 12 bytes for the nonces, 4 bytes for the source identifier, and one bit for the message type ACK (hence less that 250 bytes).

\subsection{Symbolic Analysis of SR3}

We conducted a symbolic analysis of SR3, focusing on authentication, using the tool Scyther [15]. Overall, the symbolic analysis focuses more on the protocol than on the cryptography, because of few key differences with the previous section. 
First, this analysis is done in the symbolic model instead of computational model. This model assumes the perfect encryption hypothesis, which specifies that cryptographic primitives are perfect black-boxes, and the attacker can only interact with them through the expected properties: for instance, the attacker can decrypt $\mathrm{E}_{k}(x)$ if and only if he has knowledge of $k$. This knowledge is built from a DolevYao model [16], which specifies that the attacker only knows what can be built or deduced from the communications he overhears.

The symbolic model is more restrictive for an attacker than the computational model. Here, we still use the description given in Figure 4 (page 11), but we consider an independent source node. Also, instead of proving security properties for a single session of the protocol, we determine whether the attacker can execute a bounded number of sessions of the protocol to achieve its goal. The attacker can alter, delete, or create messages, based on its current knowledge, and it can also initiate new protocol sessions.

We focus on authentication, more precisely non-injective agreement for both participants. This property is defined in the hierarchy given in [28]. Consider two actors, A and B, running a protocol. If the protocol verifies this property, it guarantees that if A completes a run of the protocol, apparently with B, then B has previously been running the protocol, apparently with A, and both A and B agreed on the same data (in our case, this data is both Data and $N$ ).

We model the authenticated encryption by decomposing an Encrypt-then-MAC primitive into two parts: an encryption and a MAC (Message Authentication Code) part. Our modeling for Scyther is available online [4] and we experimented it setting the bound on the number of sessions to 100. Under this condition, Scyther reported that SR3 provides the aforementioned authentication property for both actors A and B.

\section{Experimental Results}

In this part, we evaluate our protocol with respect to classical measures, namely, delivery rate of the messages, fairness, and number of hops. We also study the resiliency of SR3 against several attack scenarios. For that purposes, we ran simulations using Sinalgo [34], an event-driven simulator for WSNs, and we compared the performances of SR3 to those of six other routing protocols.

\subsection{Experimental Conditions}

We deployed sensors uniformly at random on a square plane. We positioned the sink at the center of the square plane. The compromised nodes are selected uniformly at random among other sensors. Two nodes can communicate if and only if their Euclidean distance is less or equal to a preset fixed range, i.e., the topology is a Unit Disk Graph (UDG). We only considered connected topologies. The communication links are asynchronous and FIFO. To enforce asynchronism (i.e., to maximize interleavings of events), the transmission time of each link follows an exponential random distribution of parameter $\lambda=1$ (so, the average transmission time is 1). Only honest sensors generate data to route. The time between two 
consecutive data generations at the same sensor also follows an exponential random distribution, whose parameter $\lambda$ is the same for all sensors and whose value depends on the average degree $\bar{\delta}$ and the number of sensors $n$ in the network, to prevent congestion, namely $\lambda=\frac{\sqrt{\bar{\delta}}}{10 n}$.

If we fix the number of nodes $n$ and the range of the UDG, we can tune the size of the simulation area to control the expected average degree $\bar{\delta}$ of the network. In our simulations, $n$ varies from 50 to 400 and $\bar{\delta}$ varies from 8 to 32 . The percentage of compromised nodes varies from 0 to $30 \%$. We considered various attack scenarios, where compromised nodes made selective forwarding: each compromised node drops received messages with a probability $p \in(0,1]$ (if $p=1$, the node is called a blackhole). In addition, some compromised nodes may have some additional "bad" skills, e.g., they may be wormholes or Sybil. A compromised node is said to be Sybil when it pretends to be multiple, distinct nodes in the system. A wormhole is a compromised node, typically far from the sink, which (temporarily) violates the UDG topology by directly communicating (via a fast private medium) with the sink in order to attract the traffic.

For each setting (number of nodes, average degree, attack scenario, amount of compromised nodes, and routing algorithm), we ran simulations over 20 UDGs, randomly generated. In each simulation, 500000 data are generated. The simulation stops once all messages have been routed or lost. We made more than 13000 simulation runs and the overall number of generated data is greater than 6 billion.

\subsection{List Sizes}

SR3 uses three lists, whose respective sizes are bounded. We made several experiments to set the size of each list to the appropriate value. Of course, the size of these three lists are influenced by the network load, which in turn is influenced by the transmission time, drop rate, and data generation time intervals (all those parameters have been set in Subsection 4.1).

We consider first the list $L_{\text {Reputation }}$. We experiment several possible values for its size, $s_{R}$. In these experiments, we implement $L_{\text {AckRouting }}$ and $L_{\text {Sent }}$ as infinite lists, which correspond to their ideal (yet impractical) behavior, to prevent any side effect.

When the behavior of malicious nodes is homogeneous over the time, the greater $s_{R}$ is, the better the delivering rate is. However, if $s_{R}$ is big, this results in increasing the time required to refresh the content of the list. So, in the case of malicious nodes that suddenly change their behavior (i.e., a sinkhole attack), the system spends more time to recover if $s_{R}$ is big. In other words, if the behavior of malicious nodes is heterogeneous with time, a large list $L_{\text {Reputation }}$ would reduce the adaptivity, and consequently the overall delivery rate, of our algorithm.

To create a sinkhole attack, we use wormholes: their ability makes them more attractive than other nodes, as they allow delivering messages faster to the sink. During the first third of the simulation, wormholes send all received data messages in their channel directly connected to the sink and route acknowledgments as honest nodes in order to obtain a high reputation. Next, they become blackholes, i.e., they drop all messages they receive.

The necessary tradeoff to obtain when choosing the value of $s_{R}$ is illustrated in Figure 6, where results are given for a network of 200 nodes, average degree 16, 


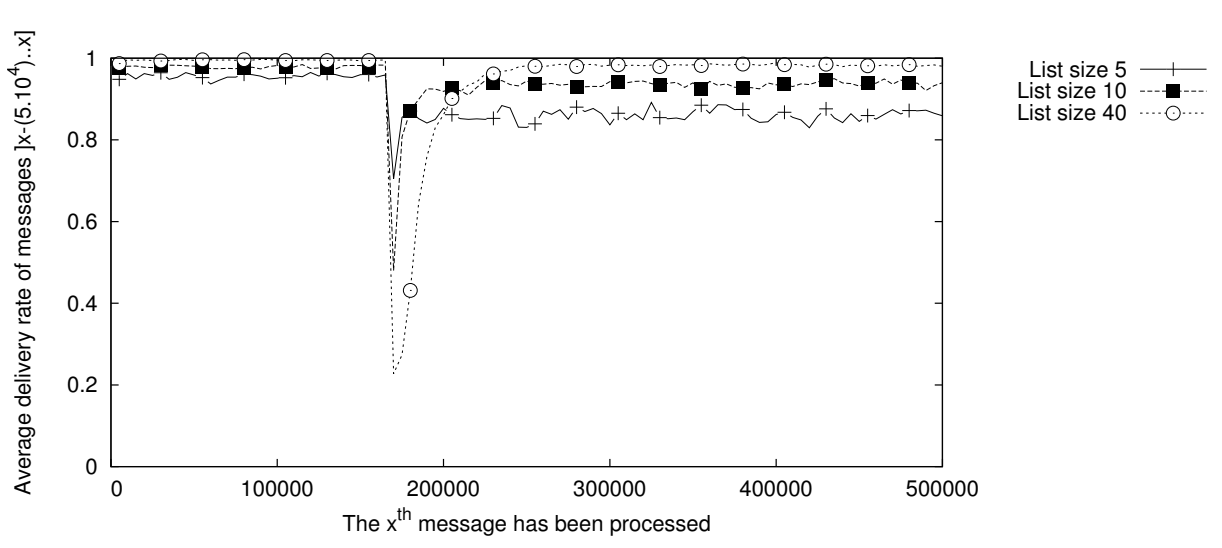

Fig. 6 Average delivery rate $(10 \%$ of $\mathrm{WH} / \mathrm{BH}, 10 \%$ of $\mathrm{BH}, n=200, \bar{\delta}=16)$

and $10 \%$ of blackholes, and $10 \%$ of wormholes/blackholes which become blackholes after the first third of the simulation. For each point $(x, y)$ of the curves, $y$ is the delivery rate computed over a window of 50000 messages, from the $(x-50000)^{t h}$ to the $x^{\text {th }}$ emitted message.

We test the aforementioned scenario with several values for $s_{R}$ - from 5 to 40. For each size, we run simulations on networks of sizes 100, 200, and 400 with average degrees 8,16 , and 32 , and containing both $10 \%$ of blackholes $(\mathrm{BH})$ and $10 \%$ of wormholes/blackholes $(\mathrm{WH} / \mathrm{BH})$. We made 10 simulations (each with a different topology) for each setting (list size, number of nodes, degree). The overall results are summarized in Table 1 . In each cell of the table, we print the value of $s_{R}$ that offers the best average delivery rate.

\begin{tabular}{cc||c|c|c} 
& \multicolumn{1}{c||}{} & \multicolumn{3}{c}{ Number of nodes } \\
& & 100 & 200 & 400 \\
\hline \hline \multirow{2}{*}{ Average degree } & 8 & 10 & 5 & 5 \\
\cline { 2 - 5 } & 16 & 15 & 10 & 5 \\
\cline { 2 - 5 } & 32 & 20 & 15 & 5
\end{tabular}

Table 1 Best observed $L_{\text {Reputation }}$ size $s_{R}$, when facing $10 \% \mathrm{BH}$ and $10 \% \mathrm{WH} / \mathrm{BH}$.

An important fact does not appear in Table 1: the average delivery rate is very similar for a large range of list sizes. We chose $s_{R}=10$, which appeared to be a good compromise in each setting.

Next, we consider the list $L_{\text {Sent }}$. The size of both $L_{\text {Sent }}$ and $L_{\text {AckRouting }}$ are bounded for practical reasons only. Indeed, sensors have tight local memories. Now, having infinite sizes for those lists would offer the best behavior. The goal here is to find a reasonable size that achieves the adequate trade-off between performances and resource consumption.

To set the size $s_{S}$ of $L_{\text {Sent }}$, we led experiments, where $s_{R}=10$ (the value we choose previously), but where $L_{\text {Ack Routing }}$ is still implemented as an infinite list. 


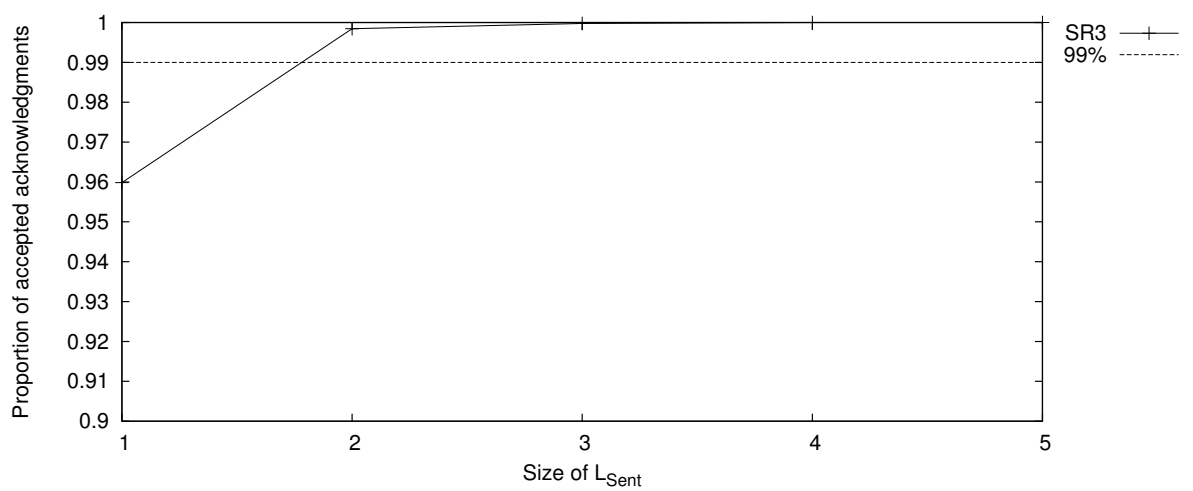

Fig. 7 Proportion of accepted acknowledgments, depending on $L_{\text {Sent }}$ size $(n=200$ and $\bar{\delta}=8)$

In those experiments, our goal is to minimize the proportion of events called false negatives. We call false negatives valid acknowledgments that return to their destination, while their corresponding nonce has been removed from $L_{\text {Sent }}$. In that case, the valid acknowledgment is simply discarded.

We made our simulations in safe networks, because this corresponds to the worst case (in terms of number of false negatives), where the routes followed by data messages and acknowledgments are longer.

We tested $s_{S}$ with values $1,3,5,7$, and 9 . The results for a particular setting is depicted in Figure 7. Actually we obtained similar results for each setting. We can see value 3 for $s_{S}$ is sufficient to reach our objective of $99 \%$ of accepted acknowledgments. Therefore, we chose to set $s_{S}$ to 3 .

Finally, we repeated the same process to set the size $s_{A}$ of $L_{\text {AckRouting }}$ using the sizes previously selected for $L_{\text {Reputation }}(10)$ and $L_{\text {Sent }}(3)$. Again, we tried to minimize the proportion of false negatives. If the list is too small, more messages would be randomly routed, which would in turn increase the delay before they reach their destination, and consequently, increase the number of false negatives. 


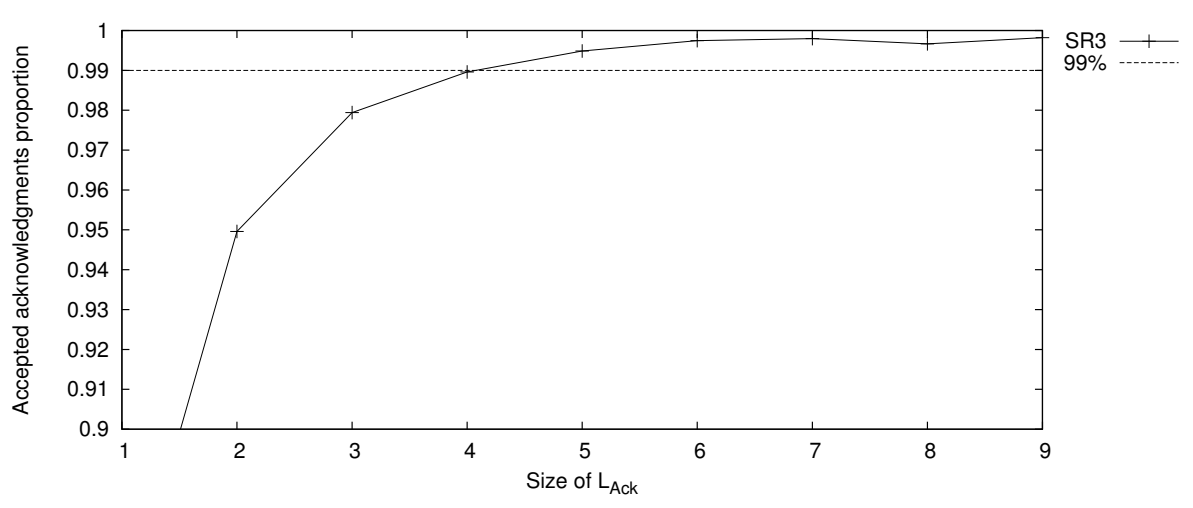

Fig. 8 Proportion of valid acknowledgments, depending on $L_{\text {AckRouting }}$ size $(n=200$ and $\bar{\delta}=8)$

Figure 8 is an example of results we obtained, using the same setting as the previous example (200 nodes, average degree 8 ). Other settings give similar results. The proportion of accepted acknowledgments stagnates from the size 5 for $L_{\text {AckRouting }}$, so we chose that value for $s_{A}$.

\subsection{Benchmark Protocols}

Resiliency is the property targeted by our experiments. It has been introduced by Erdene-Ochir et al [22]. In this latter paper, they study resiliency of some classical protocols. They propose new routing solutions dedicated to resiliency in [19]. Hence, in the following, we compare SR3 to a panel of six algorithms proposed and/or studied in these two papers [19,22].

More precisely, in [22], Erdene-Ochir et al propose a classification of routing protocols:

- Topological-based protocols that use topological information (e.g. hop distances) to deterministically determine the routes.

- Probabilistic protocols.

- Geographic protocols that determine the routes using GPS information.

- Hierarchical protocols, such as RPL [1], that split source nodes into different routing roles.

As in [22], we exclude this latter category from our panel because, in our scenario all source nodes play the same role. Again, similarly to [22], we consider one member of each of the three first categories (actually the same as those studied in $[22])$ :

1. The Gradient-Based Routing (GBR) [33] is a topological-based protocol which routes messages along a DODAG, this latter being based on hop distances and rooted at the sink. Precisely, a source node forwards all messages it receives to its (preferred) parent in the DODAG, i.e., a neighbor of lowest level in the DODAG. 
2. The Uniform Random Walk $(R W)[2]$ is a probabilistic protocol in which the message holder selects the next hop destination uniformly at random among its neighbors until the message reaches the sink.

3. The Greedy-Face-Greedy protocol (GFG) [14] is a geographic routing protocol where two modes are alternatively used: Greedy and Face. The Greedy mode (which routes according to the smallest geographic distance) is preferably used, but may lead a message to a dead end. In this case, the Face mode allows the message to escape.

Notice that GBR is close to the hierarchical protocol RPL [1], since RPL is also based on a DODAG rooted at the sink.

We also compare SR3 to the three resilient solutions proposed by ErdeneOchir et al in [19]. These solutions are respectively called $R G B R, P R G B R$, and $P R D G B R$ in the following. These three protocols are actually variants of the RGB protocol, where some uncertainty is introduced using randomness to make them less predictable by an adversary.

4. $R G B R$ uses the levels of neighbors in the DODAG: each sensor chooses next hop for each message uniformly at random among its lowest-level neighbors.

5. In PRGBR, each sensor chooses between two modes: (1) with probability 0.4 the message is routed according to RGBR; (2) with probability 0.6 the message is routed to a neighbor of same level (if no such a neighbor exists, the sensor uses mode (1)).

6. PRDGBR duplicates the message at each hop and routes the two messages independently using PRGBR. To avoid congestion, each node drops the received copies of messages it already saw.

Notice that an identical approach has been recently used to propose resilient variants of RPL [23].

\subsection{Some Scenarios and Results}

\subsubsection{Average Delivery Rate}

Figures 9-11 show the delivery rates observed in networks of average degree $\bar{\delta}=8$, 16 , and 32 , facing $30 \%$ of blackholes $(\mathrm{BH})$. The size of the networks varies from 50 to 400 nodes. (Note that, with $30 \%$ of blackholes, several honest nodes cannot safely reach the sink and consequently have delivery rate zero.) We remark that SR3 always offers a better delivery rate than the other protocols on networks of average degrees 8 and 16. In networks of average degree 32, its delivery rate is approximately the same as PRDGBR, while still better than the other protocols. In particular, the greater the networks are, the greater the gap is.

Figure 12 shows the delivery rates observed in networks of size $n=200$ facing $30 \%$ of blackholes (BH). The average degree of the networks varies from 8 to 32 . Again, we can remark that SR3 always offers the best delivery rate in that case. Moreover, as for RW and GFG, the average delivery rate of SR3 is insensitive to the degree variation. In contrast, the observed delivery rates for gradient-based protocols are low in sparse networks. In high-density networks, the performances of PRDGBR match those of SR3. However, SR3 use only two messages per data, 


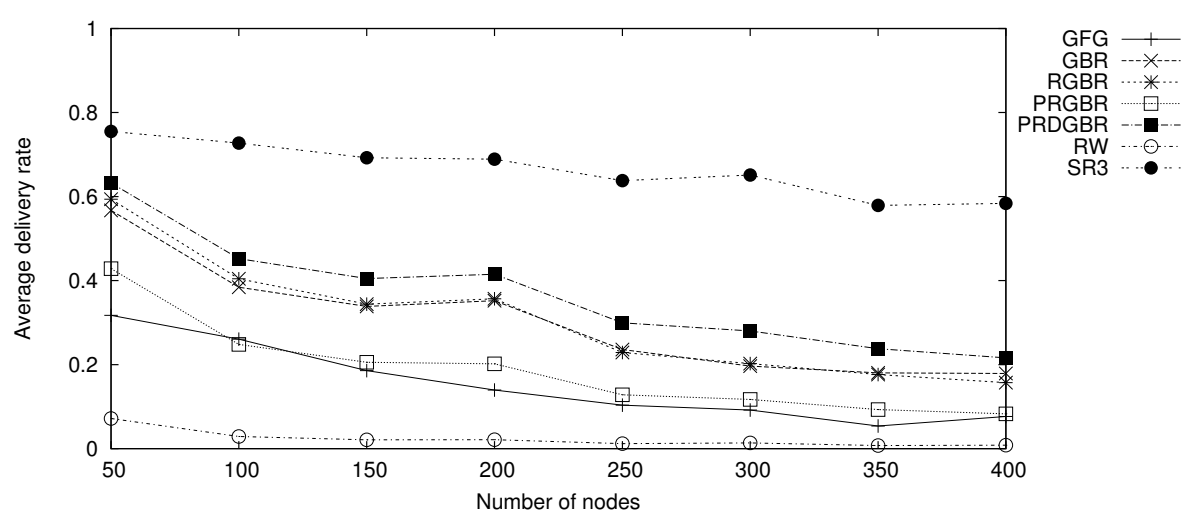

Fig. 9 Average delivery rate (30\% of $\mathrm{BH}$ nodes, $\bar{\delta}=8)$

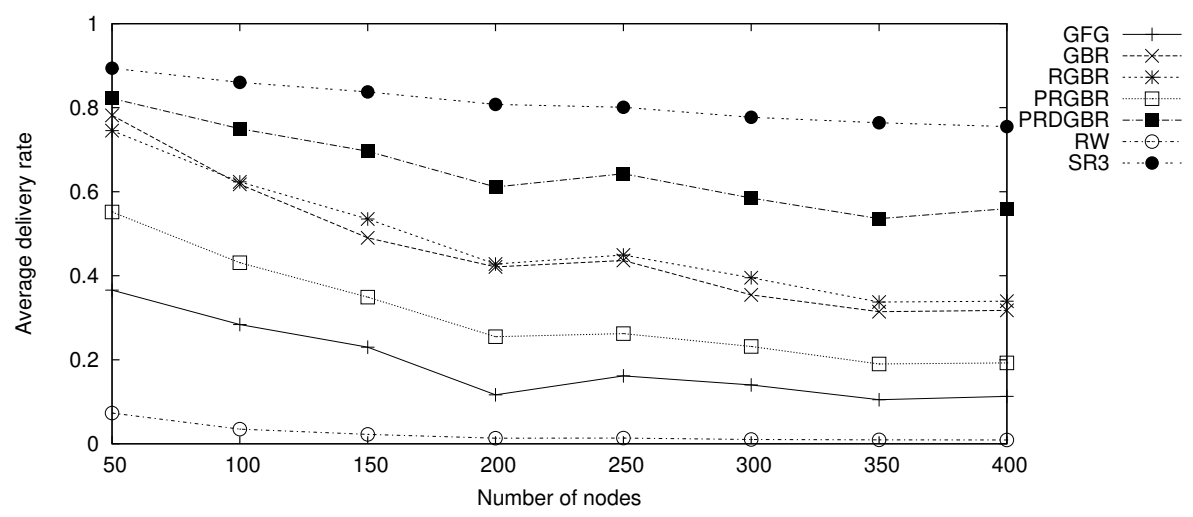

Fig. 10 Average delivery rate (30\% of $\mathrm{BH}$ nodes, $\bar{\delta}=16)$

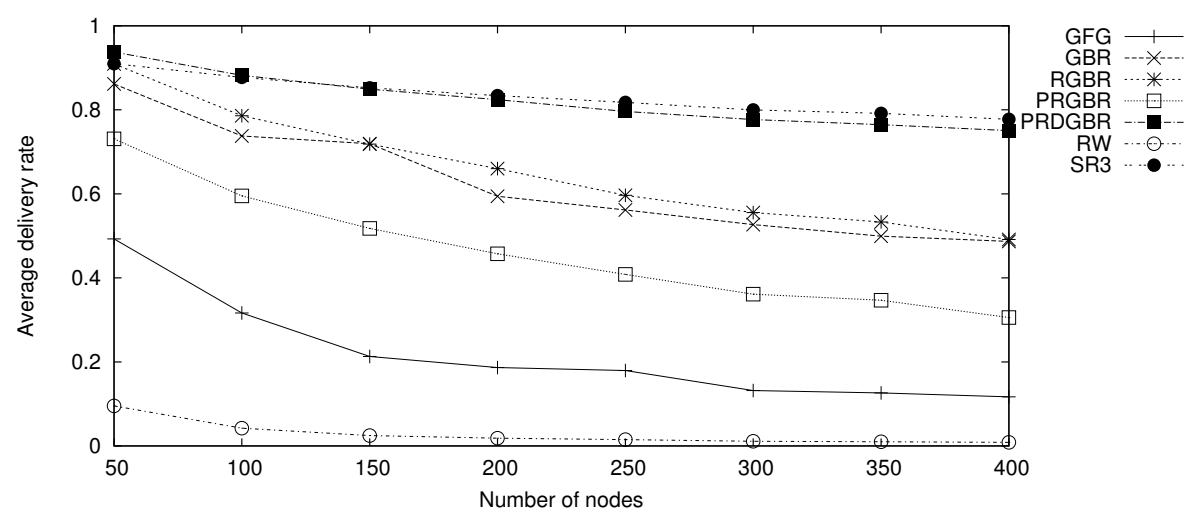

Fig. 11 Average delivery rate (30\% of $\mathrm{BH}$ nodes, $\bar{\delta}=32)$ 


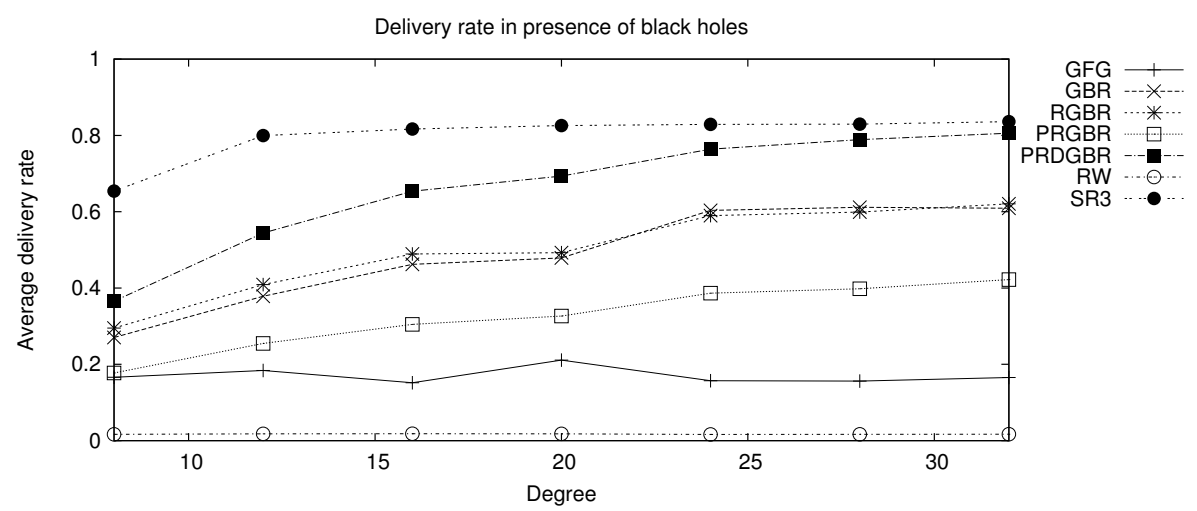

Fig. 12 Average delivery rate (30\% of $\mathrm{BH}$ nodes, $n=200)$

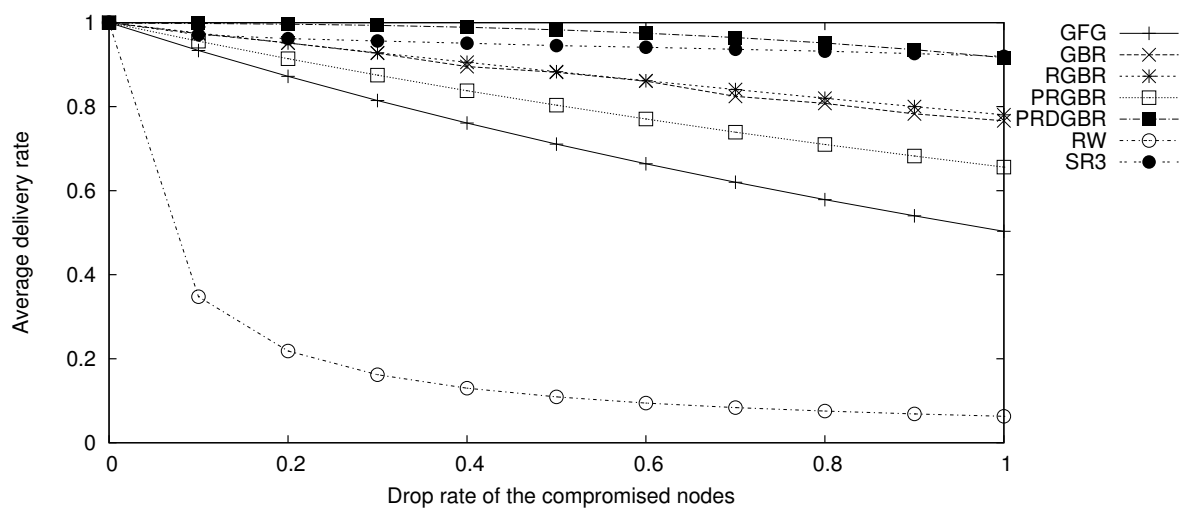

Fig. 13 Average delivery rate (20\% of SF nodes, $n=200, \bar{\delta}=8)$

while PRDGBR duplicates the messages at each hop, and consequently heavily increases the load of the network.

SR3 also efficiently combats the selective forwarding (SF) attacks. Figure 13 shows the average delivery rates observed in networks of size $n=200$ and average degree $\bar{\delta}=8$ that have to face $20 \%$ of compromised nodes, according to the drop rate of these nodes. We can observe that, except RW, all protocols of the panel achieve a graceful degradation in delivery rate when the drop rate increases. Still, SR3 offers one of the best performance. Only PRDGBR has performances close to those of SR3 when the drop rate is $100 \%$ (that is, when compromised node are actually blackholes). But again, this performance comes at the price of a high communication overhead.

We also considered networks of size $n=200$ and average degree $\bar{\delta}=8$, where $10 \%$ of nodes are both blackholes and Sybil (SY). The number of pseudonymous identifiers of these compromised nodes varies from 1 to 10 . We can observe in Figure 14, that except for GFG, adding Sybil nodes does not change the relative performances in the panel. Actually, GFG is insensitive to Sybil attacks because 


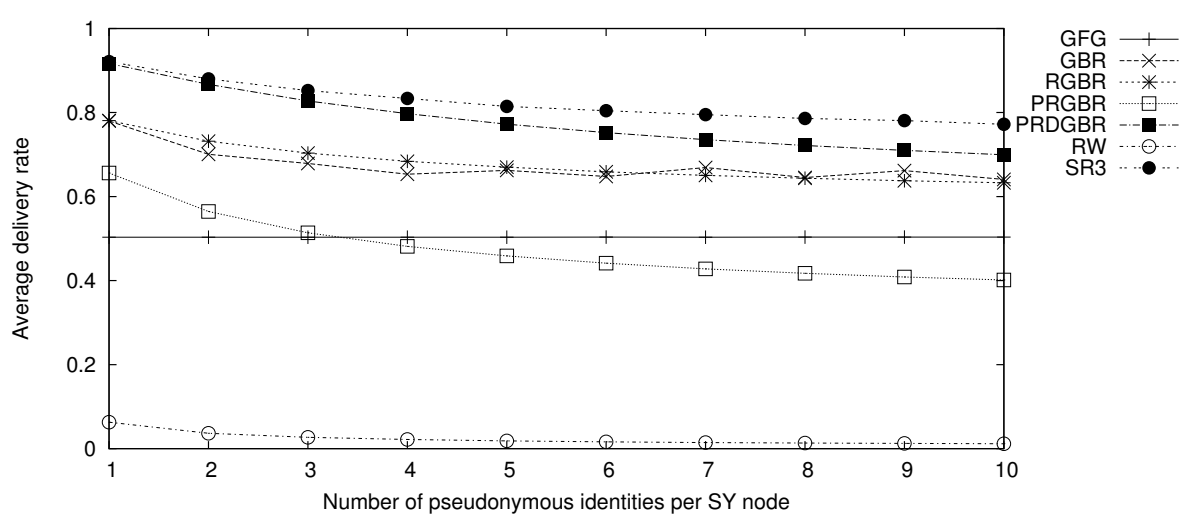

Fig. 14 Average delivery rate (10\% of SY nodes, $n=200, \bar{\delta}=8$ )

\begin{tabular}{c||c|c} 
Algorithm & Average delivery rate & Standard deviation \\
\hline GFG & 0.117 & 0.308 \\
\hline GBR & 0.487 & 0.487 \\
\hline RGBR & 0.491 & 0.307 \\
\hline PRGBR & 0.306 & 0.223 \\
\hline PRDGBR & 0.750 & 0.179 \\
\hline RW & 0.008 & 0.017 \\
\hline SR3 & 0.777 & 0.060 \\
\hline
\end{tabular}

Fig. 15 Average delivery rate and standard deviation of the delivery rate of nodes (30\% of $\mathrm{BH}, n=200, \bar{\delta}=32$ )

it does not use node identifiers. Now, still in that case, SR3 offers the best performances.

\subsubsection{Fairness}

Fairness among the delivery rates of honest nodes is a desired property in routing protocols. A classical way to capture this property is to compute the standard deviation of the delivery rates of honest nodes [20]. Figure 15 shows the average and standard deviation of delivery rates observed in networks of size $n=200$ and average degree $\bar{\delta}=32$, when facing $30 \%$ blackholes. The smaller the standard deviation is, the fairer the algorithm is. Now, a shortcoming of this measure is that when the delivery rates are uniformly bad (like for example in RW), the observed fairness is good. So, analyzed alone, this measure is misleading.

Instead, we propose here to visualize the distributions of delivery rates. Figure 16 shows an example of our method. In this figure, we consider the same simulations as in Figure 15. There is one column per algorithm of the panel. Each column represents the range of possible delivery rates from 0 to $100 \%$, by intervals of $10 \%$. The color shade encodes the proportion of nodes having the corresponding delivery rate. Consider, for example, the RW protocol: almost all nodes have a delivery rate of less than $10 \%$. In contrast, using SR3, almost all nodes have a delivery rate greater or equal to $70 \%$. We can clearly observe two classes of processes when looking at GFG and GBR: nodes have either $0 \%$ or $100 \%$ of delivery 


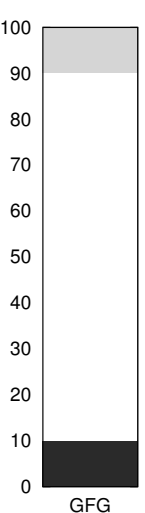

Proportion of nodes (\%):
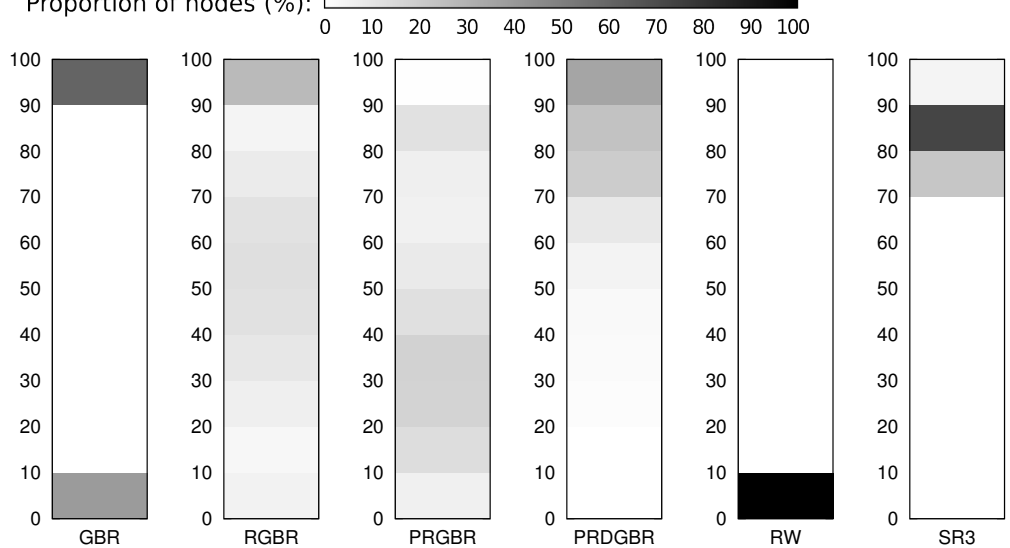

Fig. 16 Average delivery rate distribution ( $30 \%$ of $\mathrm{BH}, n=200, \bar{\delta}=32$ )

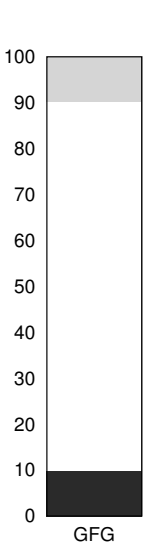

Proportion of nodes (\%)
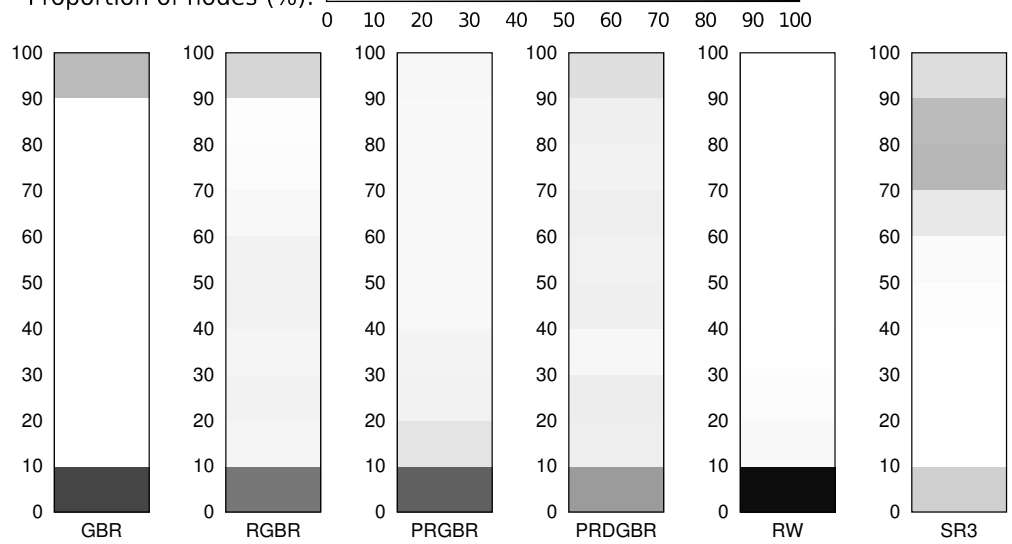

Fig. 17 Average delivery rate distribution (30\% of $\mathrm{BH}, n=200, \bar{\delta}=8$ )

rate; these protocols are unfair. The probabilistic variants of GBR are fairer: the delivery rates are spread on the whole range, but still these results are weaker than those observed for SR3.

We also provide other results in Figure 17. Simulations were run on networks of size $n=200$ with an average degree $\bar{\delta}=8$, also when facing $30 \%$ blackholes. Overall, we observe results similar to the previous setting, with a few differences. First, GBR and the variants have overall lower deliver rate and fairness, as they behave better in highly connected networks. Also, in this special case, there are more nodes which lost all of their messages, as there is no guarantee for the existence of safe paths for all sources. 


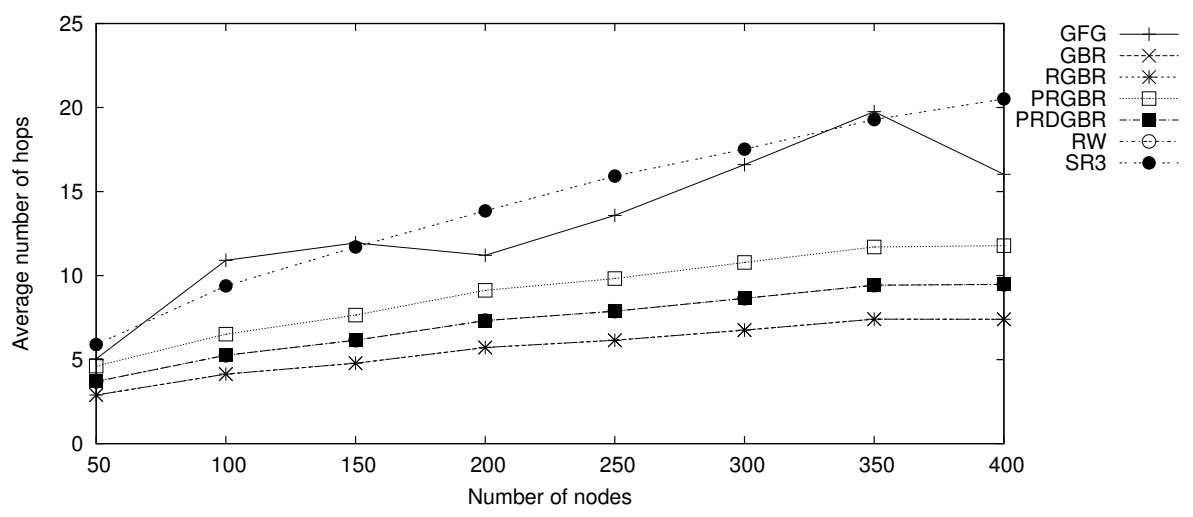

Fig. 18 Average number of hops in safe networks $(\bar{\delta}=8)$

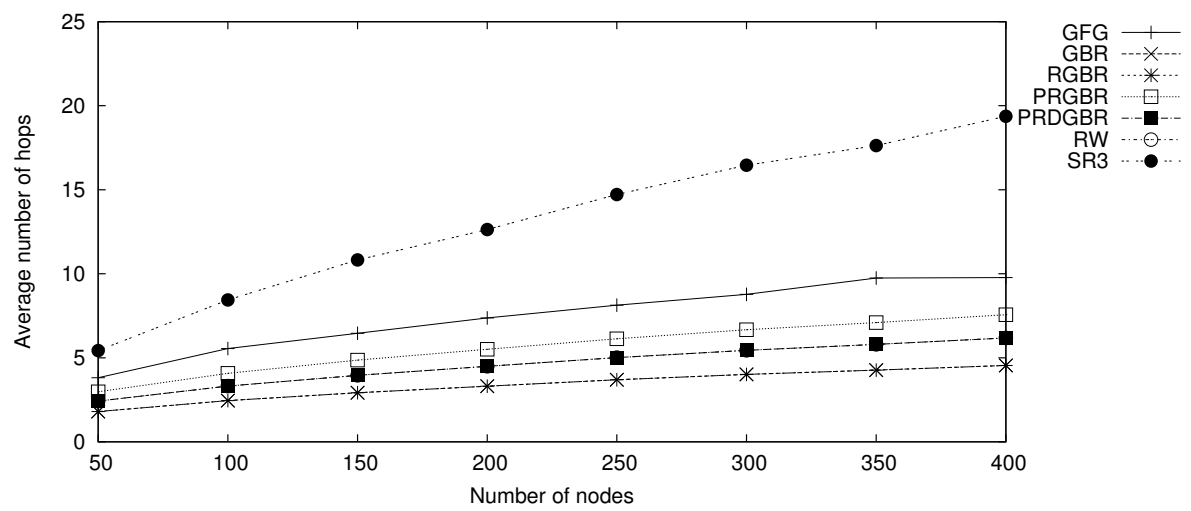

Fig. 19 Average number of hops in safe networks $(\bar{\delta}=16)$

\subsubsection{Average Number of Hops}

Here, we are only interested in the messages that are successfully delivered. So, we consider safe networks. Figures 18-20 show the average number of hops of data messages in networks of average degree respectively $\bar{\delta}=8,16$ and 32, where the size $n$ varies from 50 to 400 . First, note that we do not show results for RW in the figure because they are drastically worse than other protocols of the panel, e.g., for 50 nodes and $\bar{\delta}=16$, its average number of hops is 40 , and for 400 nodes and $\bar{\delta}=16$, its average number of hops is 529 . Then, by definition, routes followed using GBR or RGBR are optimal. Finally, SR3 generates longer routes than the geographical and gradient-based protocols due to its lack of knowledge about the network. However, this length stays reasonable (i.e. we always observed lengths drastically smaller than $n$ ), and scales with the number of nodes. Note that Greedy-Face-Greedy does not behave well in some low-degree graphs, this is due to the existence of dead ends in those graphs. 


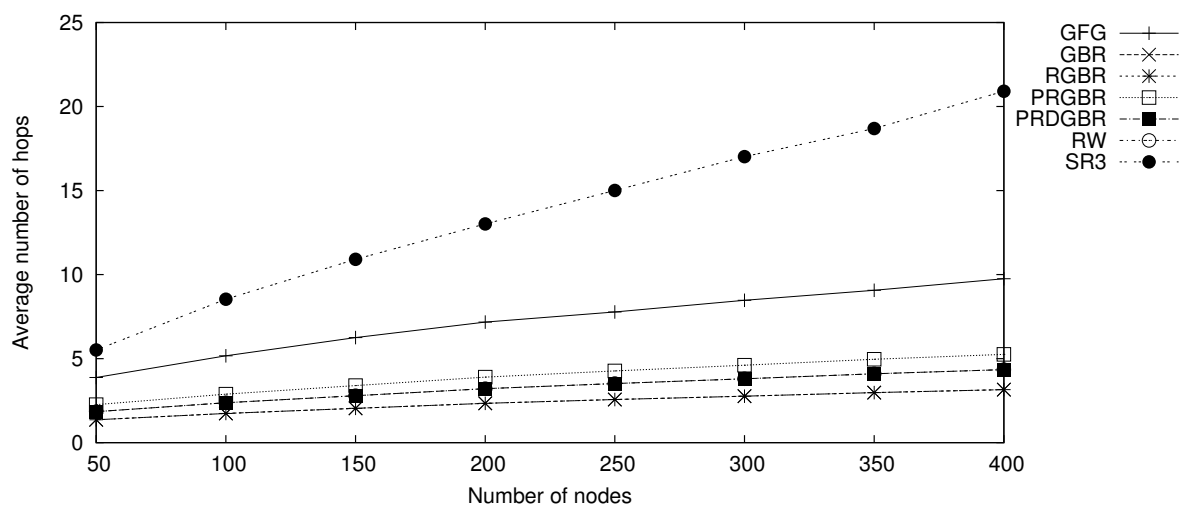

Fig. 20 Average number of hops in safe networks $(\bar{\delta}=32)$

\subsubsection{Self-adaptativity}

Thanks to its reputation mechanism, SR3 self-adapts to the variations of the hostile environment. To see this, consider the following scenario: in a network of $n=$ 200 nodes with average degree $\bar{\delta}=8$, we assume $5 \%$ of blackholes and $5 \%$ of wormholes/blackholes $(\mathrm{WH} / \mathrm{BH})$, that first behave as wormholes to attract the traffic, and then become blackholes after one third of the simulation. Such nodes appear more attractive to their neighbors because they allow delivering messages faster. Figure 21 shows the evolution of the delivery rates of each protocol: for each point $(x, y)$ of the curves, $y$ is the delivery rate computed over a window of 10000 messages, from the $(x-10000)^{t h}$ to the $x^{t h}$ emitted message. Only SR3 recovers from this attack.

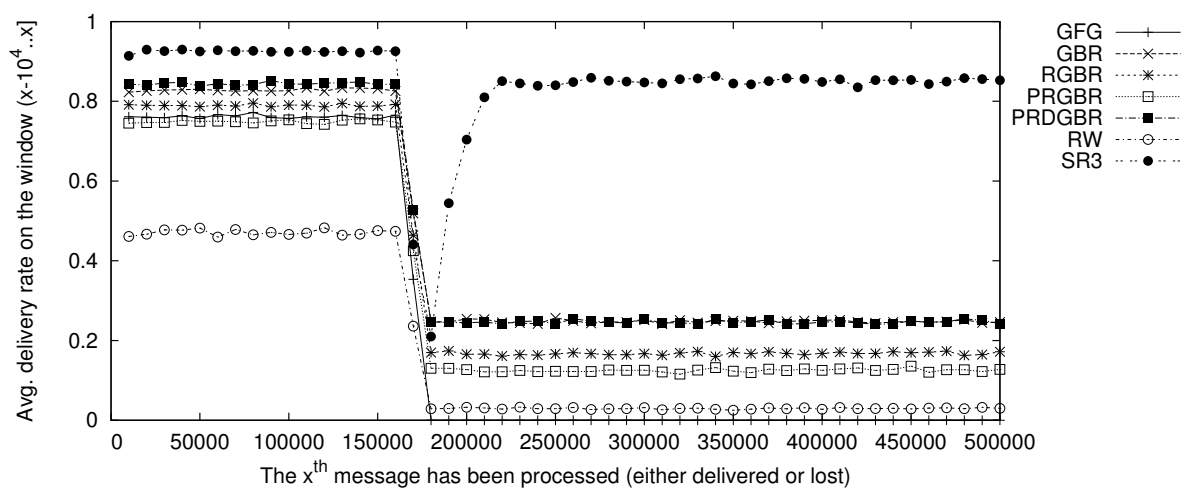

Fig. 21 Average delivery rate ( $5 \%$ of $\mathrm{WH} / \mathrm{BH}, 5 \%$ of $\mathrm{BH}, n=200, \bar{\delta}=8)$

We show in Figure 22 the average delivery rate distribution in the same setting as previously. We see that the deterministic protocols cause nodes to deliver either 
none, one third or all their messages. The randomization used in the GBR variants causes a more spread out distribution, but there is still a large concentration of nodes delivering between 30 and 40 percent of their messages. Finally, as observed before, SR3 is fair: most nodes deliver more than 70 percent of all their messages, whereas for the other protocols managing to deliver some data, the delivery rates of the nodes in this scenario strongly depend on their position in the network.

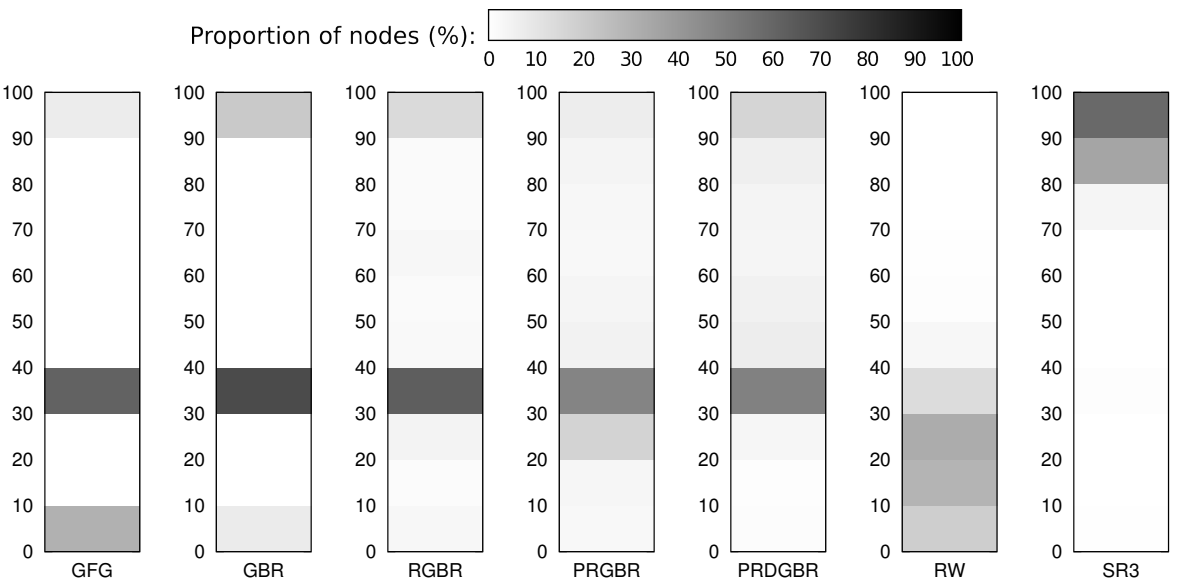

Fig. 22 Average delivery rate distribution ( $5 \%$ of $\mathrm{WH} / \mathrm{BH}, 5 \%$ of $\mathrm{BH}, n=200, \bar{\delta}=8$ )

\section{Concluding Remarks}

We proposed SR3, a secure and resilient algorithm for convergecast routing in wireless sensor networks. Using lightweight cryptographic primitives, SR3 achieves data confidentiality and unforgeability. Using simulations, we showed the resiliency of SR3 in various attack scenarios, including selective forwarding, blackhole, wormhole, and Sybil nodes. The comparative study shows that the resiliency accomplished by SR3 is drastically better than the one achieved by several routing protocols of the literature, even those whose targeted metric is resiliency.

The immediate perspective of this work is to study the performances of SR3 in a more dynamic environment, e.g., networks with mobile nodes or networks where nodes are added/removed on the fly.

An implementation of SR3 in a WSN testbed platform is currently being finalized, the preliminary results are promising, and it should be ready soon for real-world experimentations.

\section{Acknowledgments}

The authors are grateful to Bruno Blanchet for his meticulous reading of the paper and his numerous suggestions. 


\section{References}

1. Accettura, N., Grieco, L., Boggia, G., Camarda, P.: Performance analysis of the RPL routing protocol. In: Mechatronics (ICM), 2011 IEEE International Conference on, pp. $767-772(2011)$

2. Aleliunas, R., Karp, R., Lipton, R., Lovasz, L., Rackoff, C.: Random walks, universal traversal sequences, and the complexity of maze problems. In: 20th Annual Symposium on Foundations of Computer Science, pp. 218-223 (1979)

3. Altisen, K., Devismes, S., Jamet, R., Lafourcade, P.: SR3: Secure resilient reputation-based routing. In: Distributed Computing in Sensor Systems (DCOSS), 2013 IEEE International Conference on, pp. 258-265 (2013). DOI 10.1109/DCOSS.2013.33

4. Altisen, K., Devismes, S., Jamet, R., Lafourcade, P.: SR3 supplementary material (2013). URL http://www-verimag.imag.fr/ devismes/SR3/

5. Bellare, M.: Symmetric encryption. https://cseweb.ucsd.edu/ mihir/cse207/w-se.pdf

6. Bellare, M.: New proofs for nmac and hmac: Security without collision-resistance (2006). URL https://cseweb.ucsd.edu/ mihir/papers/hmac-new.html. An extended abstract of this paper appeared in Advances in Cryptology - Crypto 2006 Proceedings, Lecture Notes in Computer Science Vol. 4117, C. Dwork ed, Springer-Verlag, 2006.

7. Bellare, M., Canetti, R., Krawczyk, H.: Keying hash functions for message authentication. In: Proceedings of the 16th Annual International Cryptology Conference on Advances in Cryptology, CRYPTO '96, pp. 1-15. Springer-Verlag, London, UK, UK (1996). URL http://dl.acm.org/citation. cfm?id=646761.706031

8. Bellare, M., Kilian, J., Rogaway, P.: The security of the cipher block chaining message authentication code. J. Comput. Syst. Sci. 61(3), 362-399 (2000). DOI 10.1006/jcss.1999. 1694. URL http://dx.doi.org/10.1006/jcss.1999.1694

9. Bellare, M., Namprempre, C.: Authenticated encryption: Relations among notions and analysis of the generic composition paradigm. J. Cryptol. 21(4), 469-491 (2008). DOI 10.1007/s00145-008-9026-x. URL http://dx.doi.org/10.1007/s00145-008-9026-x

10. Bellare, M., Rogaway, P.: Random oracles are practical: A paradigm for designing efficient protocols. In: Proceedings of the 1st ACM conference on Computer and communications security, pp. 62-73 (1993)

11. Bellare, M., Rogaway, P.: Encode-then-encipher encryption: How to exploit nonces or redundancy in plaintexts for efficient cryptography. In: T. Okamoto (ed.) Advances in Cryptology - ASIACRYPT 2000, 6th International Conference on the Theory and Application of Cryptology and Information Security, Kyoto, Japan, December 3-7, 2000, Proceedings, Lecture Notes in Computer Science, vol. 1976, pp. 317-330. Springer (2000). DOI 10.1007/3-540-44448-3_24. URL http://dx.doi.org/10.1007/3-540-44448-3_24

12. Blanchet, B.: A computationally sound mechanized prover for security protocols. IEEE Trans. Dependable Sec. Comput. 5(4), 193-207 (2008)

13. Bogdanov, A., Khovratovich, D., Rechberger, C.: Biclique cryptanalysis of the full aes. In: Advances in Cryptology-ASIACRYPT 2011, pp. 344-371. Springer (2011)

14. Bose, P., Morin, P., Stojmenović, I., Urrutia, J.: Routing with guaranteed delivery in ad hoc wireless networks. Wireless Networks 7(6), 609-616 (2001)

15. Cremers, C.J.: The scyther tool: Verification, falsification, and analysis of security protocols. In: Computer Aided Verification, pp. 414-418. Springer (2008)

16. Dolev, D., Yao, A.: On the security of public key protocols. Information Theory, IEEE Transactions on $\mathbf{2 9}(2), 198-208$ (1983)

17. Ehrsam, W., Meyer, C., Smith, J., Tuchman, W.: Message verification and transmission error detection by block chaining (1978). URL http://www. google. com/patents/US4074066. US Patent 4,074,066

18. Eisenbarth, T., Kumar, S.: A survey of lightweight-cryptography implementations. Design \& Test of Computers, IEEE 24(6), 522-533 (2007)

19. Erdene-Ochir, O., Kountouris, A., Minier, M., Valois, F.: Enhancing resiliency against routing layer attacks in wireless sensor networks: Gradient-based routing in focus. International Journal On Advances in Networks and Services 4(1 and 2), 38-54 (2011)

20. Erdene-Ochir, O., Kountouris, A.A., Minier, M., Valois, F.: A new metric to quantify resiliency in networking. IEEE Communications Letters 16(10), 1699-1702 (2012). DOI 10.1109/LCOMM.2012.081612.121191. URL http://dx.doi.org/10.1109/LCOMM. 2012 . 081612.121191

21. Erdene-Ochir, O., Minier, M., Valois, F., Kountouris, A.: Resiliency of wireless sensor networks: Definitions and analyses. In: Telecommunications (ICT), 2010 IEEE 17th International Conference on, pp. 828-835 (2010) 
22. Erdene-Ochir, O., Minier, M., Valois, F., Kountouris, A.: Toward resilient routing in wireless sensor networks: Gradient-based routing in focus. In: Proceedings of the 2010 Fourth International Conference on Sensor Technologies and Applications, SENSORCOMM '10, pp. $478-483$ (2010)

23. Heurtefeux, K., Erdene-Ochir, O., Mohsin, N., Menouar, H.: Enhancing RPL resilience against routing layer insider attacks. In: L. Barolli, M. Takizawa, F. Xhafa, T. Enokido, J.H. Park (eds.) 29th IEEE International Conference on Advanced Information Networking and Applications, AINA 2015, Gwangju, South Korea, March 24-27, 2015, pp. 802-807. IEEE Computer Society (2015)

24. Hu, Y., Perrig, A., Johnson, D.: Ariadne: A secure on-demand routing protocol for ad hoc networks. Wireless Networks 11(1-2), 21-38 (2005)

25. Katz, J., Yung, M.: Unforgeable encryption and chosen ciphertext secure modes of operation. In: G. Goos, J. Hartmanis, J. van Leeuwen, B. Schneier (eds.) Fast Software Encryption, Lecture Notes in Computer Science, vol. 1978, pp. 284-299. Springer Berlin Heidelberg (2001). DOI 10.1007/3-540-44706-7_20. URL http://dx.doi.org/10.1007/ 3-540-44706-7_20

26. Koblitz, N.: Elliptic Curve Cryptosystems. Mathematics of Computation 48(177), 203-209 (1987)

27. Liu, D., Ning, P.: Multilevel tesla: Broadcast authentication for distributed sensor networks. ACM Transactions in Embedded Computing Systems (TECS) 3, 800-836 (2004)

28. Lowe, G.: A hierarchy of authentication specifications. In: Computer Security Foundations Workshop, 1997. Proceedings., 10th, pp. 31-43. IEEE (1997)

29. Miller, V.S.: Use of elliptic curves in cryptography. In: Advances in Cryptology CRYPTO 85 Proceedings, vol. 218, pp. 417-426 (1986)

30. Papadimitratos, P., Haas, Z.: Secure Routing for Mobile Ad hoc Networks. In: Proceedings of the SCS Commnication Networks and Distributed Systems Modeling and Simulation Conference (CNDS), pp. 193-204 (2002)

31. Perrig, A., Szewczyk, R., Tygar, J., Wen, V., Culler, D.: Spins: Security protocols for sensor networks. Wireless networks 8(5), 521-534 (2002)

32. Rackoff, C., Simon, D.R.: Non-interactive zero-knowledge proof of knowledge and chosen ciphertext attack. In: J. Feigenbaum (ed.) Advances in Cryptology - CRYPTO '91, 11th Annual International Cryptology Conference, Santa Barbara, California, USA, August 11-15, 1991, Proceedings, Lecture Notes in Computer Science, vol. 576, pp. 433444. Springer (1991). DOI 10.1007/3-540-46766-1_35. URL http://dx.doi.org/10.1007/ 3-540-46766-1_35

33. Schurgers, C., Srivastava, M.: Energy efficient routing in wireless sensor networks. In: Proceedings of MILCOM 2001, pp. 357-361 (2001)

34. Sinalgo: Simulator for network algorithms. http://www.disco.ethz.ch/projects/sinalgo/. Distributed Computing Group at ETH Zurich

35. Ye, W., Heidemann, J.S., Estrin, D.: Medium access control with coordinated adaptive sleeping for wireless sensor networks. IEEE/ACM Trans. Netw. 12(3), 493-506 (2004). DOI 10.1109/TNET.2004.828953. URL http://dx.doi.org/10.1109/TNET.2004.828953 